\title{
A Study on the Essential and Parkinson's Arm Tremor Classification ${ }^{\dagger}$
}

\author{
Vasileios Skaramagkas ${ }^{1} \mathbb{D}$, George Andrikopoulos ${ }^{2, * \mathbb{D}}$, Zinovia Kefalopoulou ${ }^{3}$ and \\ Panagiotis Polychronopoulos 3 (D)
}

1 Institute of Computer Science, Foundation for Research and Technology Hellas (FORTH), 70013 Heraklion, Crete, Greece; vskaramag@ics.forth.gr

2 Computer Science, Electrical and Space Engineering Deptartment, Luleå University of Technology, 97187 Luleå, Sweden

3 Neurology Department, Patras University Hospital, 26504 Patras, Greece; zkefalopoulou@gmail.com (Z.K.); ppolychr@yahoo.gr (P.P.)

* Correspondence: geoand@ltu.se; Tel.: +46-920-492-451

$+\quad$ This paper is an extended version of our paper published in 28th Mediterranean Conference on Control and Automation (MED 2020), Saint-Raphaël, France, 15-18 September 2020.

Citation: Skaramagkas, V.;

Andrikopoulos, G.; Kefalopoulou, Z.; Polychronopoulos, P. A Study on the Essential and Parkinson's Arm Tremor Classification. Signals 2021, 2 , 201-224. https://doi.org/10.3390/ signals2020016

Academic Editor: Giorgos Giannakakis

Received: 5 November 2020

Accepted: 15 April 2021

Published: 19 April 2021

Publisher's Note: MDPI stays neutral with regard to jurisdictional claims in published maps and institutional affiliations.

Copyright: (C) 2021 by the authors. Licensee MDPI, Basel, Switzerland. This article is an open access article distributed under the terms and conditions of the Creative Commons Attribution (CC BY) license (https:// creativecommons.org/licenses/by/ $4.0 /)$.

\begin{abstract}
In this article, the challenge of discriminating between essential and Parkinson's tremor is addressed. Although a variety of methods have been proposed for diagnosing the severity of these highly occurring tremor types, their rapid and effective identification, especially in their early stages, proves particularly difficult and complicated due to their wide range of causes and similarity of symptoms. To this goal, a clinical analysis was performed, where a number of volunteers including essential and Parkinson's tremor-diagnosed patients underwent a series of pre-defined motion patterns, during which a wearable sensing setup was used to measure their lower arm tremor characteristics from multiple selected points. Extracted features from the acquired accelerometer signals were used to train classification algorithms, including decision trees, discriminant analysis, support vector machine (SVM), K-nearest neighbor (KNN) and ensemble learning algorithms, for providing a comparative study and evaluating the potential of utilizing machine learning to accurately discriminate among different tremor types. Overall, SVM related classifiers proved to be the most successful in terms of classifying between Parkinson's, essential and no tremor diagnosed with percentages reaching up to $100 \%$ for a single accelerometer measurement at the metacarpal area. In general and in motion while holding an object position, Coarse Gaussian SVM classifier reached $82.62 \%$ accuracy.
\end{abstract}

Keywords: essential tremor; Parkinson's tremor; classification; machine learning

\section{Introduction}

A tremor [1] is defined as the involuntary rhythmic oscillation of a part of the human body, which is produced by the competing acting contractions of the muscles in the joints. The need to find the tremor's origin and its diagnosis is imperative to achieve immediate reduction and treatment of the related syndrome. Tremor is distinguished in the following basic categories: (a) resting tremor, where the affected body part is placed on a surface so that it remains unaffected by the force of gravity, (b) postural tremor, which is the most commonly occurring type, where the subject attempts to keep his trembling body part steady during a pose affected by gravity and (c) motion tremor, which occurs during movement and increases with its duration [2].

There are several tremor types, each with different causes and characteristics: Parkinsonian (Parkinson's disease and parkinsonism), essential, cerebellum, post-traumatic, pharmaceutical, dystonia, multiple sclerosis, neuropathy, Holmes, Wilson disease, psychogenic tremor, etc. [3]. The clinical picture and the proper reception of medical history, as 
well as the experience of the individual doctor contribute to a more accurate determination of tremor type. From the different types of tremor, essential and Parkinson's are described by the highest occurrence, reaching up to $5 \%$ and $1 \%$ of a country's population [4], respectively, and with increasing frequency. Despite the existence of various diagnostic methods for Parkinson's and tremor, their rapid and effective discrimination, especially in their early stages, proves particularly difficult, mainly due to their wide range of causes and similarity of symptoms [5].

The challenge of finding an accurate and effective method for discriminating among tremor types, while being able to predict their stage and severity, has led to the use of sensorenabled systems for extracting various elements of tremor, which cannot be extracted solely from the clinical picture of the patient. Recently, there has been an increasing interest of applying machine and deep learning modeling methodologies on the various acquired bio-signals for identifying and predicting the severity of Parkinson's disease [6-8].

Specifically, machine learning has been used for symptom quantification of Parkinson's as well as essential tremor patients via gyroscope and accelerometer sensors [9-15]. Furthermore, recognition of Parkinson's was recently tested on phonation and speech datasets for identifying dysphonia signs related to the syndrome [16-20]. Predictive diagnosis of Parkinson's has also been attempted via machine learning on demographic, movement and speech data [21], as well as using typing and mouse behavior for detecting and monitoring the severity of Parkinson's disease with supervised machine learning classifiers [22,23]. Other bio-data including dopamine transporter data from tomographic images [24] and serum cytokines [25] have also been used for classification performance evaluation via extraction of shape features based on generated areas of interest from images and other indicators, while discrimination of patient motor status via machine learning has also been recently tested on a digital biomarker data set using Neural Network Construction methodology [26]. Deep learning modeling algorithms have also been recently utilized for tracing and identifying Parkinson's via fuzzy recurrence plots [27] and monitoring and predicting of Parkinson's disease via wearable sensors [28,29]. Work has also been done on Parkinson's diagnosis via deep learning through medical imaging [30], handwritten dynamics [31] and voice data sets [32]. Moreover, in [33], an attempt to classify between Parkinson's and essential tremor has been performed.

The aforementioned research shows the high potential in utilizing machine and deep learning for discriminating tremors. However, the majority of the studies are mainly focused on Parkinson's identification, with only a few targeting at the differential diagnosis between different tremor types [29,33-35]. Moreover, most of these efforts provide results based on a binary classification problem, while the majority utilize accelerometer sensors through wearable means from a single point of measurement, usually placed on the metacarpal region or the index finder. Such analyses are performed at postural arm poses, while the use of smartphones and their integrated accelerometers can limit the hand motion ranges. Therefore, this state-of-the-art overview shows an increasing need in further investigating this problem by expanding the classification target via inclusion of more measurement points on the user's arm, while assessed at different poses and via multiple different classifiers.

The contribution of the presented work stems from the multi-class classification problem among essential, Parkinson's tremor- and non-tremor-diagnosed volunteers. For the presented research, a clinical trial was performed, where essential and Parkinson's tremordiagnosed patients, along with healthy volunteers, underwent a series of pre-defined sequences involving multiple arm poses (resting, postural and motion patterns). During these sequences, an accelerometer-based wearable setup was used to measure their lower arm tremor characteristics from multiple selected points on the hand and forearm. Initially, the information extracted from the patients' medical history and questionnaire answers were used for assessing the efficiency of hierarchical grouping into tremor discrimination. Then, the extracted features from all acquired accelerometer signals were used as a basis for a comparative study between multiple different classification algorithms, including 
decision trees, discriminant analysis, support vector machine (SVM), K-nearest neighbor $(\mathrm{KNN})$ and ensemble learning algorithms. The goal was to provide a detailed evaluation on utilizing machine learning algorithms for accurately discriminating between the two different tremor types, while providing information on the arm measurement points that provide better classification results. It has to be noted that this work is based on the initial findings presented in [36], providing the hierarchical grouping evaluation, as well as extended performance and accuracy results on the essential and Parkinson's tremor discrimination via the multiple utilized classification algorithms.

The rest of the article is structured as follows. Section 2 provides an overview of essential and Parkinson's tremor, along with the existing diagnostic methods. Section 3 presents the measurement setup specifics utilized in the clinical trial. In Section 4, the methodology concerning the data processing algorithms for patient categorization, feature extraction, training and prediction are provided in detail. Section 5 provides the results of the clinical trial, along with commentary on the acquired data involving the volunteer categorization, hierarchical grouping and tremor classification. Finally, concluding remarks along with comments on future work are provided in Section 6.

\section{Essential and Parkinson's Tremor}

\subsection{Essential Tremor: Overview and Diagnosis}

Essential tremor (ET) is described as a disorder of the nervous system in which the hands and arms are usually trembling [37]. Individuals over 40 years of age who are diagnosed with essential tremor belong to $4 \%$ of the world's population [38]. Some of them manifest tremor only in certain postures (static shaking), while others present it when performing a task, for example during writing or eating (motor shaking). Most patients with ET experience both shaking forms, while voluntary movements, such as holding a fork, usually worsen the tremor. This contrasts with Parkinson's disease, in which the tremor tends to diminish with such movements. While the Parkinsonian tremor maximizes at rest with respect to amplitude and frequency [39], the essential tremor's higher frequency and amplitude occur mainly during activity and are decreased at rest [40]. However, these changes in the tremor amplitude and frequency cannot be easily determined and depend each time on the affected person and on the condition stage. The causes of ET are various genetic mutations, which are not yet fully determined, and it is often observed that ET patients have difficulty in daily activities, such as writing, swallowing food, speaking, etc. [41]. Before someone is diagnosed with ET, other factors must first be excluded, such as:

- chronic alcohol use;

- intake of antiarrhythmic, antidepressant and antispasmodic drugs;

- use of substances such as nicotine or cocaine.

Tremor can additionally be caused by thyroid problems, strokes and Parkinson's disease. It is not uncommon for Parkinson's Tremor (PT) to be mistaken for ET. Initially, ET usually affects the hands, head and voice, while the tremor that characterizes PT occurs primarily in the hands and then spreads to the jaw, legs and other parts of the body. It is also important to note that ET does not cause any other health problems, unlike Parkinson's disease that causes problems in posture, movement and gait. Finally, as it was previously stated, ET occurs mainly during movement and not in a resting position such as Parkinson's disease.

ET has some clinical traits that facilitate its diagnosis, namely that the tremor:

- Starts gradually, mainly on one side of the body and focusing on the hands.

- Gets worse as movement increases.

- Increases with caffeine intake, stress, excessive fatigue and abrupt temperature changes.

- Causes a "yes-yes" and "no-no" head movement.

In addition to the ET symptoms mentioned above, as with PT, there is a scale of tremor evaluation called The Essential Tremor Rating Assessment Scale (TETRAS) [42], presented in Table 1. TETRAS evaluates the tremor in the head, the language, the face, the voice, along 
with the upper and lower limbs. Furthermore, TETRAS rates the tremor by evaluating the performance during the conduction of daily activities, such as writing. Depending on the performance of the examined person, tremor is scored 0 to 4 points, with 0 marking absence of tremor. The length numbers (e.g., 1-3 cm) indicate the peak oscillation amplitude of the body part under evaluation.

Table 1. The Essential Tremor Rating Assessment Scale (TETRAS) [42].

\begin{tabular}{|c|c|c|c|c|}
\hline Body Part & 1 & 2 & 3 & 4 \\
\hline Face or tongue & Slightly visible & Visible & $\begin{array}{l}\text { Obvious in most } \\
\text { facial expressions }\end{array}$ & Intense, deformable \\
\hline Voice & $\begin{array}{l}\text { Slight in voices such as } \\
\text { "eee" or "aaa" }\end{array}$ & $\begin{array}{l}\text { Visible in voices } \\
\text { such as "eee" or "aaa" } \\
\text { and slight during speech }\end{array}$ & Visible during speech & $\begin{array}{l}\text { Difficulty in understanding } \\
\text { certain words }\end{array}$ \\
\hline Upper limb & Barely visible & $1-3 \mathrm{~cm}$ & $5-10 \mathrm{~cm}$ & $20 \mathrm{~cm}$ \\
\hline Lower limb & Barely visible & Visible but feckless & $5 \mathrm{~cm}$ & $5 \mathrm{~cm}$ \\
\hline Writing & Barely visible & $\begin{array}{l}\text { Visible, all words } \\
\text { can be read }\end{array}$ & $\begin{array}{l}\text { Visible, most of the } \\
\text { words can be read }\end{array}$ & No word can be read \\
\hline Dot approximation task & Barely visible & $1-3 \mathrm{~cm}$ & $5-10 \mathrm{~cm}$ & $20 \mathrm{~cm}$ \\
\hline Upright position & Barely visible & Visible but feckless & Moderate & Severe \\
\hline
\end{tabular}

\subsection{Parkinson's Tremor: Overview and Diagnosis}

Parkinson's disease [43] is one of the most common neurodegenerative diseases, with a frequency of about $1 \%$ in the population over 65 years. It is the main representative of the exopyramidal diseases and is a result of the destruction of nerve cells in the brain, resulting in incomplete production of dopamine, with consequences in unusual brain activity. In more detail, Parkinson's disease may have a genetic cause or be due to exposure to certain toxins, in the presence of lewy particles together with a-synucleons, as well as in other factors such as age and gender. The symptoms begin gradually, often with tremor occurring in one hand. PT usually occurs at rest, i.e., when the hand is not working, but remains motionless. In the early stages of the disease, it has been observed that facial expressions are reduced, hands begin to vibrate when walking and the voice becomes softer. Other symptoms may be the change in handwriting, imbalance and reduction of automated movements [44]. In addition, Parkinson's disease may be responsible for depression and emotional swings, difficulty in thinking, swallowing problems, sleep disturbances, sudden pressure drop, sexual dysfunction, etc. However, it is important to mention that Parkinson's disease is not by itself fatal. The life-threatening factor is subject to the fact that because of these symptoms, the person could cause involuntary self-harm. For example, it is possible because of the lack of balance to fall and cause serious injuries or to aspirate food to the lungs causing pneumonia and other lung diseases.

Until today, the diagnosis of Parkinson's disease is primarily based on the clinical picture of the patient [45]. More specifically, the chances of a person suffering from the disease Parkinson's increase as the following symptoms increase, the observation of which stems from daily life tasks:

- some daily activities are accomplished slower than in the past,

- balance problems,

- the letters become smaller when writing,

- tremor appears in the palm, hand, lips and legs,

- muscle stiffness,

- problems when walking (confused feet, step diminishes),

- the feeling that the feet are stuck on the floor,

- one hand remains motionless when walking,

- change in voice and sound pitch,

- difficulty of the person to get up from a seated position,

- weakness when fastening a garment. 
At this point, a reasonable question is whether the specific symptoms are found to be capable of deciding whether the patient is suffering from Parkinson's disease. The answer to the above question is not easy and even depends on two key factors. First and decisive factor is the stage of the disease. Often in its early stages, PT can be confused with the syndrome of ET. Other times the pharmaceutical administration of anti-psychotic drugs to combat depression or even treatment of drugs to cure nausea cause PT. In addition, the syndromes of progressive supranuclear palsy [46]) and the normal pressure Hydrocephalus [47] cause symptoms similar to Parkinson's disease. The second factor relies on the particular neurologist, who initially attempts to identify the fundamental traits of the disease (tremor at rest, stiffness, slowness and instability) and then observes the reaction of the body of the patient to anti-Parkinson's drugs.

Unfortunately the error rate in diagnosing the disease, when its symptoms are not clear touches that of $25 \%$ and often the patient seeks the opinion of more than one specialist, which creates the need for an accurate diagnosis of PT. In 2011, Food and Drug Administration (FDA) approved the Datscan technique, which allows physicians to observe the dopamine system in the brain through detailed imaging. This technique is not enough to diagnose the disease, but it is an important tool when used in conjunction with the clinical picture of the patient and helps distinguishing between PT and ET. In addition, the exploitation of a Positron Emission tomography (PET) Scan offers physicians the opportunity to study the function of cells of a part of the body, in this case the brain. A different method for identifying the disease is CT-Scan [48]. During this method a radioactive drug is placed intravenously and depicts the D2 dopaminergic receptors leading to the separation of the ET from Parkinson's disease.

It is easily understood that the diagnosis of Parkinson's disease has several difficulties and depends to a large extent on the severity of the disease, as well as the medical doctor's experience. For this reason, Movement Disorder Society (MDS) created the Unified Parkinson's Disease Rating Scale (UPDRS), which consists of 4 sections and a total of 50 multiple choice questions associated with the motor and non-motor symptoms of the disease. The first two following sections are conducted by the patient, while the other two are by the expert:

- daily life experiences,

- daily life kinetic experiences,

- motion examination,

- difficulties occurring during various movements.

The acquired questionnaire replies are interpreted based on the Parkinson's disease stages model [49] presented in Table 2 and the daily activities scale [50] presented in Table 3.

Table 2. Hoen and Yahr Parkinson's disease stage model [49].

\begin{tabular}{ll}
\hline Disease Stage & Description \\
\hline Stage 0 & No sign of the disease \\
Stage 1 & Unilateral disease \\
Stage 1.5 & Unilateral disease plus axial involvement \\
Stage 2 & Bilateral illness, without any impairment of balance \\
Stage 2.5 & Mild bilateral disease, with recovery in the helix test \\
Stage 3 & Mild to moderate bilateral disease. Some volatility, independent \\
Stage 4 & Severe disabilities. Still able to walk or stand without help \\
Stage 5 & Use of wheelchair or lying in bed, unless assisted \\
\hline
\end{tabular}


Table 3. Schwab and England daily activities scale [50].

\begin{tabular}{ll}
\hline Percentage & Description \\
\hline $100 \%$ & Completely independent. Able to do all tasks without slowness or difficulty. \\
$90 \%$ & Completely independent. Able to do all tasks with some degree of slowness or difficulty. \\
$80 \%$ & Fully independent in most tasks, but with many tasks taking twice as long to complete. Awareness of \\
& difficulty and slowness. \\
$70 \%$ & Not completely independent. More challenges during some activities. \\
$60 \%$ & Some dependency. Ability to complete most tasks, but extremely slowly and with great effort. \\
$50 \%$ & More dependent. Difficulty during most tasks. \\
$40 \%$ & Very dependent. Ability to assist only in certain tasks. \\
$30 \%$ & More effort to accomplishing a few tasks. Greater assistance is needed. \\
$20 \%$ & Inability of performing unsupervised tasks. \\
$10 \%$ & Fully dependent. \\
$0 \%$ & Functions such as swallowing, cystic bladder and intestine do not work. Bedridden. \\
\hline
\end{tabular}

\section{Data Acquisition Setup}

\subsection{Setup Specifics}

In order to investigate the discrimination possibility of essential and Parkinson's tremor, as well as the effect of utilizing tremor data from different parts of the hand, a signal acquisition device was developed based on the use of 4 accelerometers for enabling the extraction of hand tremor information from multiple measurement points.

Specifically, the index and thumb were selected for their tactile dominance and kinematic difference, while the metacarpal and forearm were chosen to further analyze the tremor origin and its propagating properties. Thus, the accelerometers are placed each on the index, the thumb, the outer side of the metacarpal region and on the lower part of the volunteer's forearm, as graphically shown in Figure 1, while their placement is properly stabilized via a protective glove and the use of Velcro straps. The standard coordinate systems are defined as presented in Figure 1, where the accelerometers are placed on the outer side of the hand and parallel to the transverse plane formed when the hand is in the extended position.

To be able to extract the necessary data for classifying and discriminating PT and ET, an acquisition device was developed for implementing the aforementioned conceptual approach. The device (Figure 2) consists of 4 ADXL345 3-axes accelerometers connected to two Arduino Mega Boards via SPI protocol. The reason for utilizing two boards was for ensuring the highest possible sampling rate during the tremor recording sessions, enough for safely measuring the maximum PT and ET frequencies. Double-sided Velcro straps are used for easy and comfortable adjustment of the accelerometers on the volunteer's hand, while minimizing the effect on the measurements during hand motions and maintaining the tactile ability of the person's fingertips.

\subsection{Acquisition Procedure}

Prior to the measurement trials, all volunteers were informed about the process in detail and were asked to sign an ethical agreement form. A brief questionnaire with basic personal information (gender, age, etc.), as well as tremor-related medical information (age of first tremor occurrence, medication status, etc.) was filled, with the presence and confirmation of a supervising neurologist in the case of tremor-diagnosed patients.

Next, and for the purpose of the measurement trials, the volunteers had the setup properly set on their hand while, as defined via the utilized examination protocol, they were being asked to have the target hand at the following positions presented at Figure 3a-d:

- Resting position: The palm touches the table without exercising force (Figure 3a).

- Extended position: The hand is extended at shoulder height (Figure 3b).

- Free motion: The hand performs an oscillatory movement from the table to the nose. This movement is repeated for the whole recording at moderate speed (Figure 3c). 
- Motion while holding an object: The hand makes the same movement as in the free motion position, but this time the hand is holding a bottle half-full of water (Figure 3d).

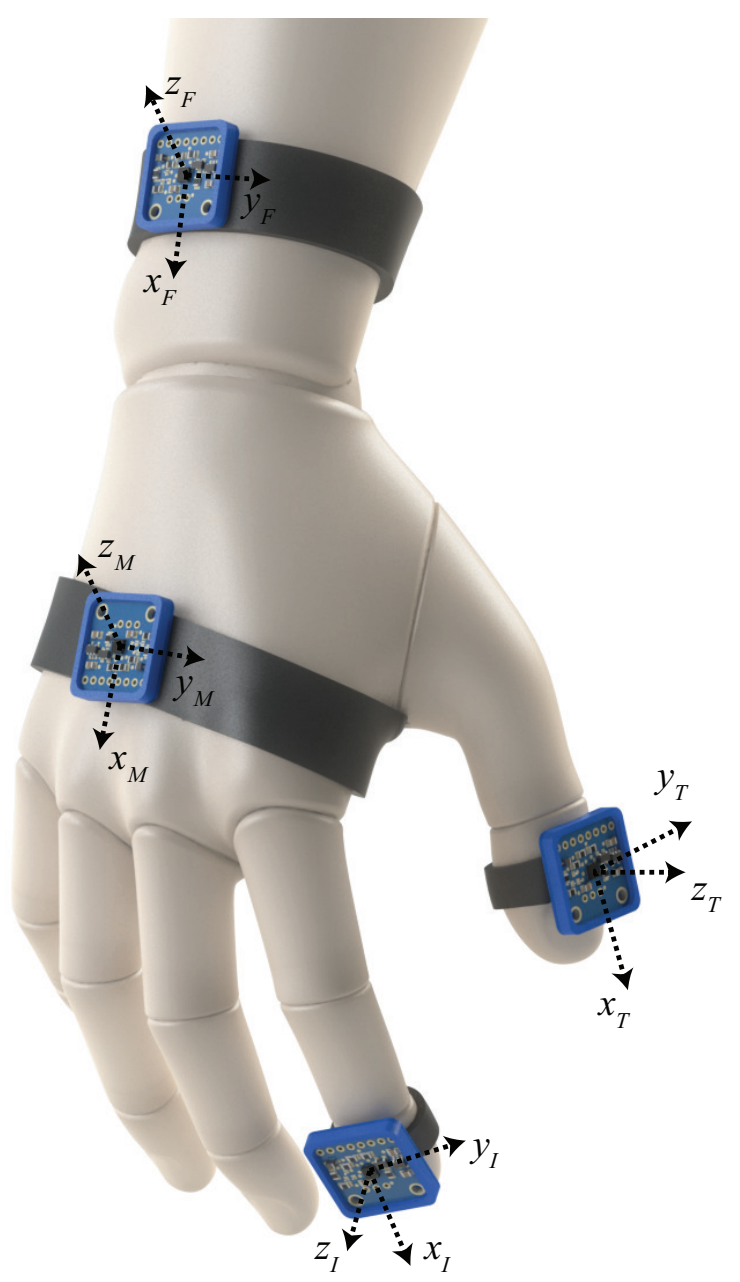

Figure 1. Graphical representation of the selected accelerometer placement points on the human hand.

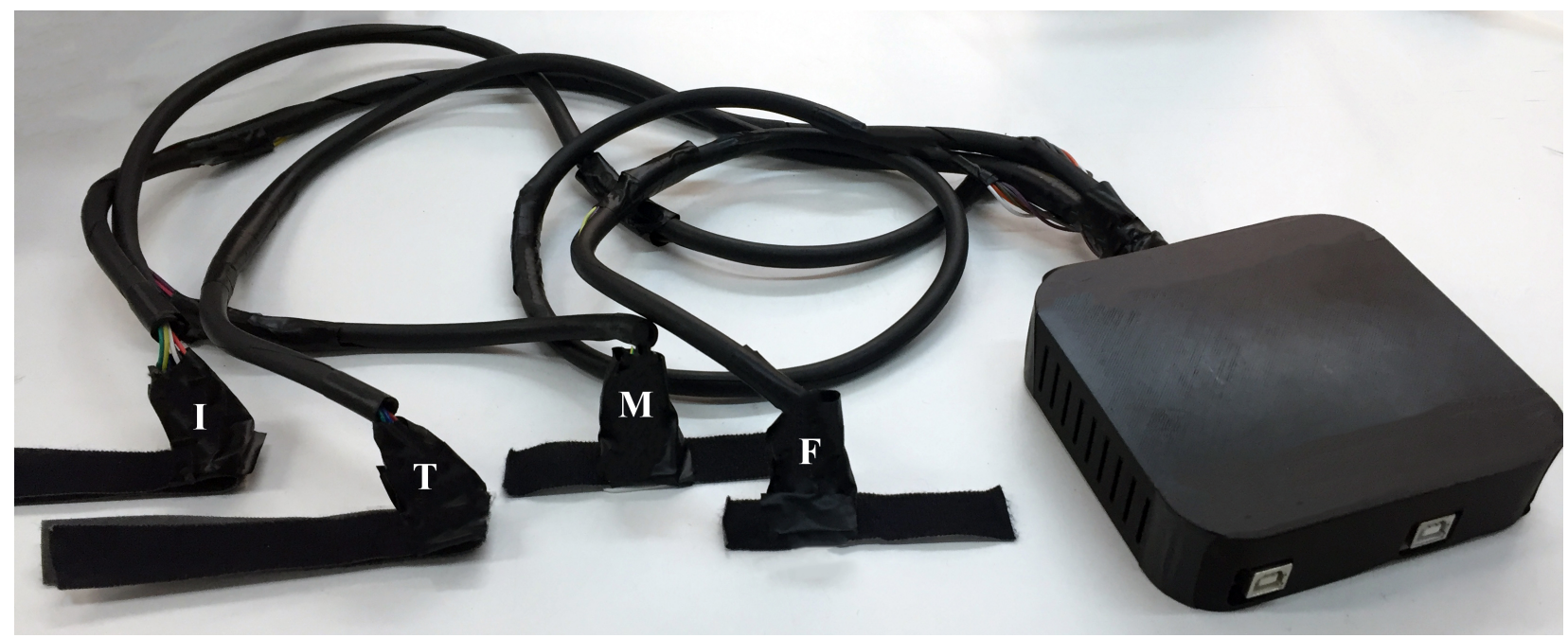

Figure 2. The measurement acquisition device utilized in the clinical trials with highlighted accelerometers $\{I, T, M, F\}=\{$ index, thumb, metacarpal, forearm $\}$. 
The duration of each measurement was $30 \mathrm{~s}$. Before the start of each measurement, it was explained to the volunteers the exact hand pose or movement that they had to perform for the duration of the recording. In case of measurement failure due to volunteer's weakness to keep his hand straight or in motion, software/hardware issue or other external factor the measurement was performed again when the problem was resolved.

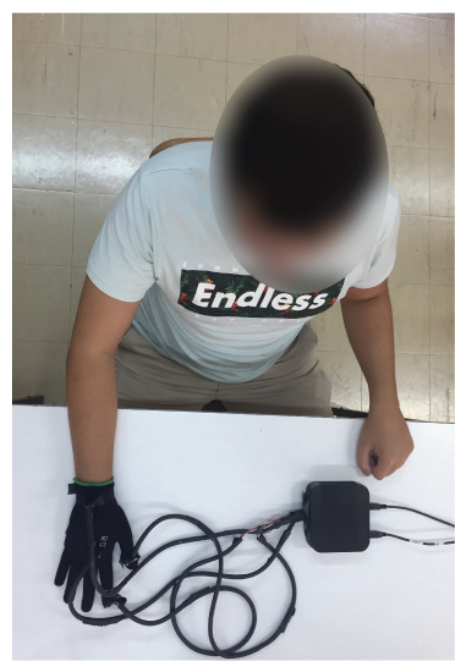

(a)

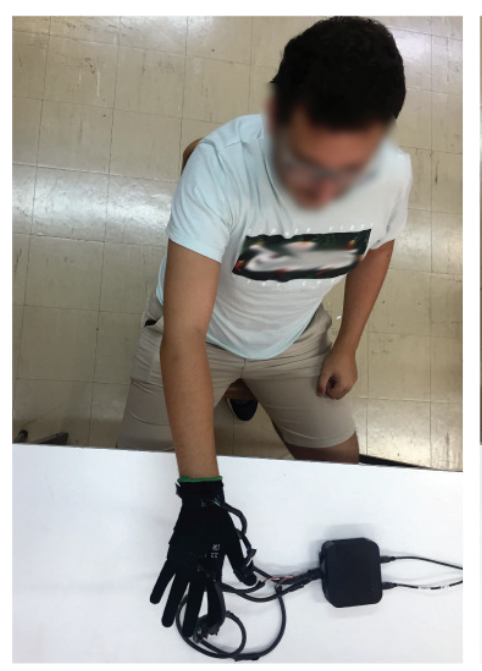

(b)

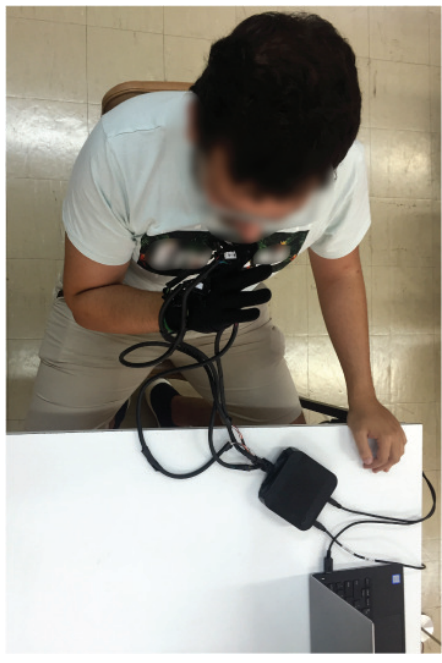

(c)

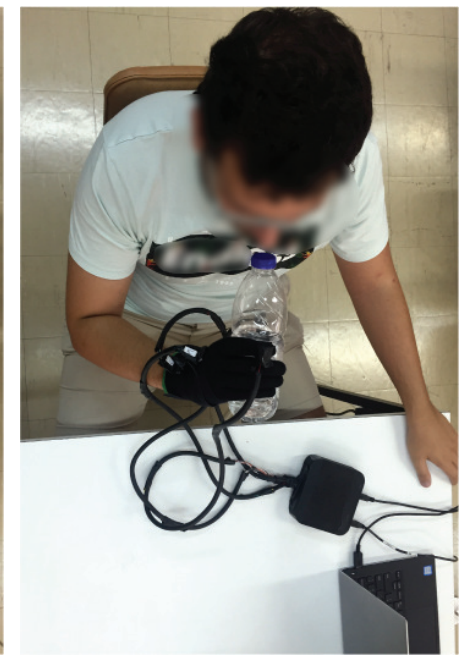

(d)

Figure 3. The selected arm poses: (a) resting position, (b) extended position, (c) free motion, (d) motion while holding an object.

\section{Methodology \\ 4.1. Data Acquisition and Processing}

The data set acquired from each volunteer is divided into 4 measurement sets for each of the $i=\{1,2,3,4\}$ arm poses: resting position $(i=1)$, extended position $(i=2)$, free motion $(i=3)$ and motion with object $(i=4)$. Every measurement set consists of 4 acquired subsets $j=\{I, T, M, F\}$, one for every accelerometer positioned on the volunteer index $(j=I)$, thumb $(j=T)$, metacarpal $(j=M)$ and forearm $(j=F)$, as summarized in Table 4 for each arm pose $i$. Each subset contains 3 acceleration signals, one for each axis $k=\{x, y, z\}$ as defined in Figure 1. Therefore, the data set acquired from each volunteer consists of a total 48 acquired acceleration signals defined as $a_{i, j, k}$ for each measurement set $i$, accelerometer position $j$ and accelerometer axis $k$.

Table 4. Measurement points for selected arm poses.

\begin{tabular}{ccccc}
\hline Position & Index & Thumb & Metacarpal & Forearm \\
\hline Rest & $I_{1}$ & $T_{1}$ & $M_{1}$ & $F_{1}$ \\
Postural & $I_{2}$ & $T_{2}$ & $M_{2}$ & $F_{2}$ \\
Free motion & $I_{3}$ & $T_{3}$ & $M_{3}$ & $F_{3}$ \\
Motion with object & $I_{4}$ & $T_{4}$ & $M_{4}$ & $F_{4}$ \\
\hline
\end{tabular}

All measurements acquisitions were performed with respect to the Nyquist criterion to avoid undersampling. The maximum sampling rate achieved for each accelerometer was 62.5 Hz, enough to safely measure the maximum tremor frequencies of PT and ET. Each measurement consisted of approximately 1800 samples, taking into account the duration of each recording. The data were then post-processed, while for each accelerometer and measuring position the frequency spectrum was calculated for extracting the dominant tremor frequency. 


\subsection{Extracted Features}

For each acquired acceleration magnitude, 12 features are extracted based on statistical signal characteristics (SSC) proposed in [33]. For extracting these features, the following procedure is utilized:

1. Division of initial acquired signal in sections $\left(N_{s}\right)$. Given that the total number of samples for each measurement is approximately 1800 (sampling rate: $62.5 \mathrm{~Hz}$ and recording time: $30 \mathrm{~s}$ ) and each section consists of 40 samples, the total number of sections is approximately 45 with no overlaps.

2. Calculation of local maximum for every section $\left(\alpha_{n}\right)$.

3. Calculation of an amplitude change, $A_{n}$, for every section, defined as the absolute difference of the maximum acceleration of the previous section $\left(\alpha_{n-1}\right)$ from the maximum of the current section $\left(\alpha_{n}\right)$, with $\alpha_{0}=0$.

$$
A_{n}=\left|\alpha_{n}-\alpha_{n-1}\right|
$$

4. Calculation of the mean amplitude change.

$$
\text { mean_amp }=\sum_{m=1}^{N_{s}} \frac{A_{m}}{N_{s}}
$$

5. Calculation of the mean absolute deviation of amplitude changes.

$$
\mathrm{amp} \_d e v=\sum_{m=1}^{N_{s}} \frac{\mid A_{m}-\text { mean_amp } \mid}{N_{s}}
$$

6. Calculation, for every section, of the period, $T_{n}$, the difference between the time of the current section's maximum and the previous section's maximum.

$$
T_{n}=t_{n}-t_{n-1}
$$

7. Calculation of the mean period.

$$
\text { t_mean }=\sum_{m=1}^{N_{s}} \frac{T_{m}}{N_{s}}
$$

8. Calculation of the mean absolute deviation of periods.

$$
\mathbf{t} \_ \text {dev }=\sum_{m=1}^{N_{s}} \frac{\mid T_{m}-t_{-} \text {mean } \mid}{N_{s}}
$$

9. Finally, from the 4 extracted parameters, the mean, maximum, and minimum across accelerometer axes of each of the 4 features described above are calculated:

- Mean, maximum and minimum value of median value sum of each section's amplitude (mean_amp).

- Mean, maximum and minimum value of median value sum of each section's amplitude deviation (amp_dev).

- Mean, maximum and minimum value of median value sum of each section's $T_{n}$ (t_mean).

- Mean, maximum and minimum value of median value sum of each section's $T_{n}$ deviation (t_dev).

\subsection{Categorization}

All volunteers are grouped under certain categories which relate to gender, age, time period from the occurrence of disease/tremor, the stage of their condition, the medication, if any, that volunteers have followed and finally the maximum tremor frequency as extracted from the calculated frequency spectrum. 


\subsection{Hierarchical Clustering}

The extracted categories are then utilized for hierarchical clustering of the data, with the purpose of revealing any similarities between patients with Parkinson's and essential tremor. In this work, the agglomerative hierarchical clustering technique is used [51]. In this technique, initially each data point is considered as an individual cluster. In each iteration, similar clusters merge to form new, larger clusters. The hierarchical grouping to be presented is intended to distinguish individuals in basic categories in order to create a basic image of the samples collected regarding the tremor-diagnosed patients.

\subsection{Classification}

After calculating the parameters from the acquired data, the features of the training data are introduced to the 21 classifiers shown in Table 5. For the Tree classifiers, the maximum number of splits is 100 for the Fine, 20 for the Medium and 4 for the coarse tree while the split criterion is the Gini's diversity index. Moreover, the covariance structure for the discriminant analysis category is selected as full. Regarding SVMs, the Kernel scale for Linear, Quadratic and Cubic is automatic while for Fine Gaussian it is 0.87, for Medium Gaussian 3.5 and for Coarse Gaussian 14. For all the SVMs, the box constrained level is selected at 1 and the multi-class method is one-vs.-one. Furthermore, for Medium, Cosine, Cubic and Weighted KNN the number of neighbors is 10, for Fine KNN it is 1 and lastly for Coarse KNN it is chosen at 100. The distance metric is defined as Euclidean for Fine, Medium, Coarse and Weighted KNN. In parallel, Cosine and Minkowski distances are used for the Cosine and Cubic KNN classifiers, respectively. Finally, for the Ensemble learning category, the maximum number of splits is 20 for the Boosted (ensemble method: AdaBoost) and RUSBoosted (ensemble method: RUSBoost) and 134 for the Bagged trees (ensemble method: Bag). For the subspace KNN classifier, the subspace dimension is 6 and the ensemble method is the subspace one. For all the ensemble classifiers the number of learners is 30 .

Table 5. Tested classification algorithms.

\begin{tabular}{ll}
\hline Category & Algorithm \\
\hline \multirow{2}{*}{ Trees [52] } & Fine tree \\
& Medium tree \\
& Coarse tree \\
\hline \multirow{2}{*}{ Discriminant analysis [53] } & Linear discriminant analysis (LDA) \\
& Quadratic discriminant analysis (QDA) \\
\hline & Linear SVM \\
& Quadratic SVM \\
Support vector machine (SVM) [54] & Cubic SVM \\
& Fine Gaussian SVM \\
& Medium Gaussian SVM \\
& Coarse Gaussian SVM \\
\hline & Fine KNN \\
& Medium KNN \\
& Coarse KNN \\
K-nearest neighbor (KNN) [55] & Cosine KNN \\
& Cubic KNN \\
& Weighted KNN \\
\hline & Boosted trees \\
& Bagged trees \\
Ensemble learning [56] & Subspace KNN \\
& RUSBoosted trees \\
\hline
\end{tabular}




\section{Results}

In this section, the results of grouping the acquired data in various categories, as well as the discrimination results between PT, ET and no-tremor (NT) conditions will be demonstrated.

\subsection{Received Data}

A total of 43 samples were collected, 12 were from patients diagnosed with PT, 3 with ET, while the other 28 were from volunteers with no diagnosed tremor (NT). The data extracted from the measuring device include the signals of acceleration in $x, y, z$ axes as a function of time $t$ and are utilized to calculate the frequency spectrum of amplitude of the resultant acceleration vector of the measured signal for the extraction of the dominant tremor frequency. Indicatively, Figure $4 a-c$ present the data acquired via a (a) Parkinson'sdiagnosed patient, (b) an ET-diagnosed patient and (c) a volunteer without tremor evidence. The acceleration measurements refer to the extended arm position and for the index $I_{2}$. It is noted that for the amplitude spectrum plots in Figure 4 a high-pass filter was applied with a cutoff frequency of $0.5 \mathrm{~Hz}$ to avoid the DC artifact at $0 \mathrm{~Hz}$.

By observing Figure $4 a$, it is easy to distinguish the occurring PT particularly after the 16th second, when the volunteer started to tire. In this case, the maximum tremor frequency is extracted from the frequency spectrum as $5.91 \mathrm{~Hz}$. From Figure $4 \mathrm{~b}$, the main extracted ET frequency for the specific measurement is observed close to $4.93 \mathrm{~Hz}$. An observed difference from the Parkinson's patient was that the volunteer's index was trembling throughout the duration of the measurement. At the same time, the PT case was observed to have a lower maximum amplitude of the frequency spectrum, which was measured close to $0.25 \mathrm{~Hz}$, while the amplitude in the ET case was measured at $0.5 \mathrm{~Hz}$.

From Figure 4c, it is easily distinct how the volunteer keeps his arm extended without shaking significantly. However, as shown by the spectral diagrams, some small amplitude frequency peaks appear, the causes of which are attributed to the measurement noise from the accelerometer. Finally and as expected, the NT volunteer's tremor frequency shows a significantly smaller amplitude in comparison to the tremor-diagnosed volunteers.

It is important to point out that there were Parkinson's-diagnosed volunteers who showed no tremor during the measurements, which was verified in the subsequent processing of the acquired data. This is expected, as tremor may be one of the basic characteristics of the disease, but it is not a necessary and unique trait of the disease, it may not have a lasting nature and may not occur during experimentation.

\subsection{Categorization}

Table 6 contains the summarized data collected from the volunteers' medical history and questionnaire answers. PT refers to Parkinsonian tremor patients, ET to essential tremor and NT means person with no diagnosed tremor. The status $O N$ refers to a person who is taking medication and has even taken medication within the last $12 \mathrm{~h}$. Instead, in the OFF mode, the person has not taken any medicine for a $12 \mathrm{~h}$ period, but is under pharmaceutical supervision. Moreover, $33 \%$ of PT subjects were at the early stage of the disease.

Table 6. Volunteer categorization groups.

\begin{tabular}{lccc}
\hline Category & PT & ET & NT \\
\hline Gender (M/F) & $7 / 5$ & $3 / 0$ & $19 / 9$ \\
Age (range) & $47-89$ & $50-69$ & $14-76$ \\
Years since tremor appearance (range) & $1-18$ & $5-42$ & - \\
Early stage (yes/no) & $0 / 3$ & $4 / 8$ & - \\
Medication (Yes(ON/OFF)/No) & $10(7 / 3) / 2$ & $1(0 / 1) / 2$ & - \\
Tremor frequency in Hz (range) & $0-8.7$ & $0-10$ & - \\
\hline
\end{tabular}



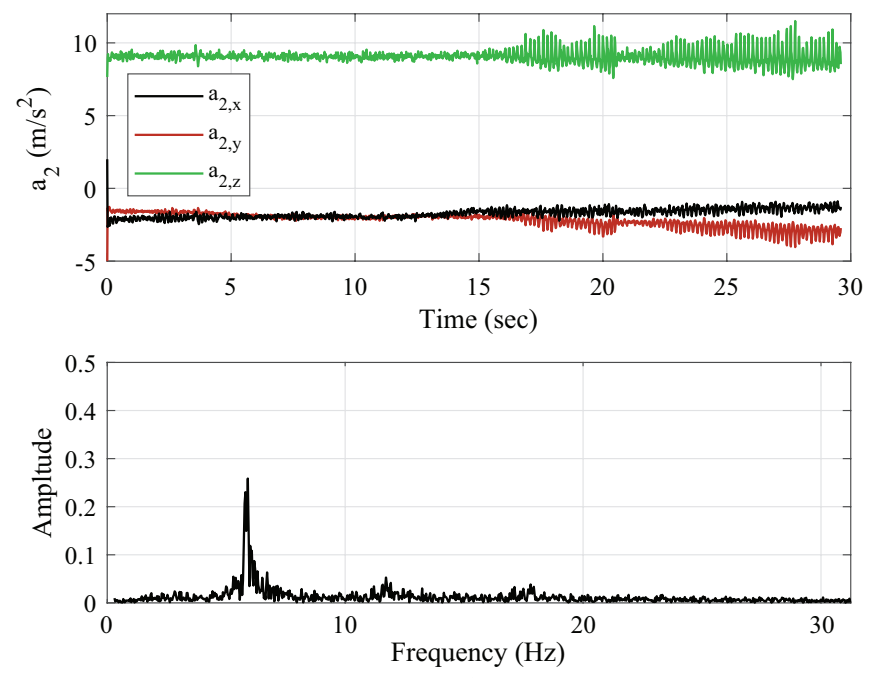

(a)
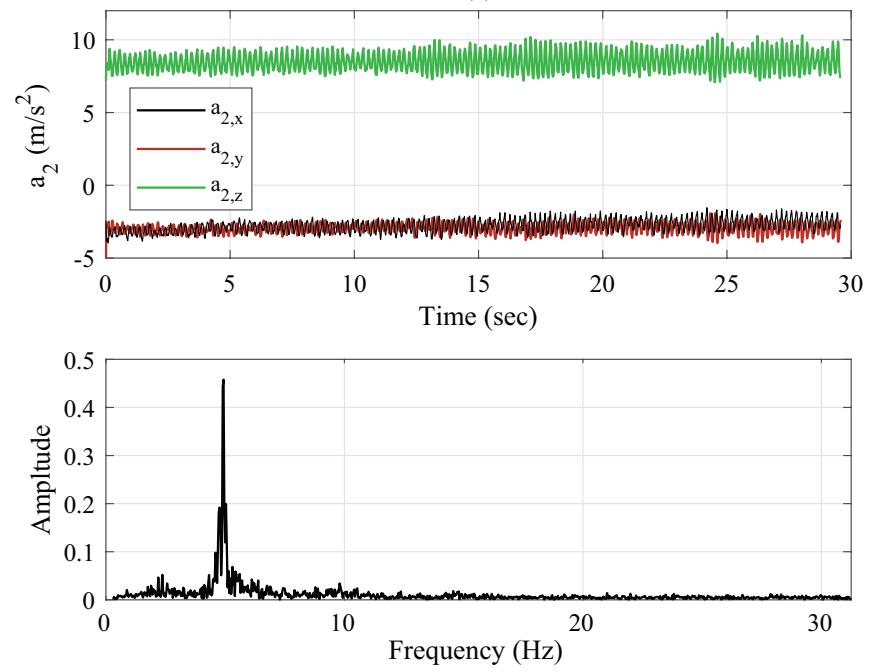

(b)
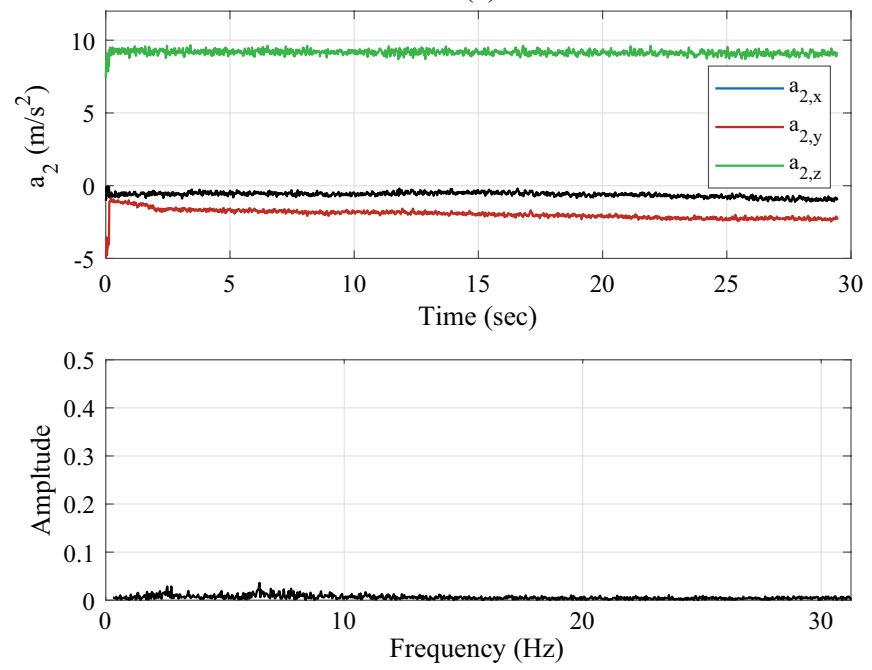

(c)

Figure 4. Indicative accelerometer measurements and respective frequency spectrum and power spectral densities, as acquired from the index measurement point and the extended arm pose $I_{2}$ of a (a) Parkinson's-diagnosed patient, (b) ET-diagnosed patient and (c) volunteer with no visible tremor evidence. 


\subsection{Hierarchical Grouping}

By considering the variables presented in Table 6, the categories of tremor, medication, gender and age are properly symbolized and normalized in the hierarchical unit, while the four classes complement the years of tremor occurrence and the maximum tremor frequency in any of the four arm poses. The indicative dendrogram produced for only the tremor-diagnosed patients (e.g., ET and PT) is presented in Figure 5. Patient numbers 1-3 (highlighted with red font) concern the volunteers with ET, while 4-15 (highlighted with green font) concern those with Parkinson's. The main remark extracted from the diagram is that the 3 volunteers with ET do not appear grouped with each other, but instead appear to have more in common with Parkinson's patients. This demonstrates the difficulty in diagnosing the tremor type, especially if that is attempted without the appropriate information, both from the part of the clinical background and from the bio-signal acquisition. Acquiring more data from volunteers with ET for extending the presented results is work in progress.

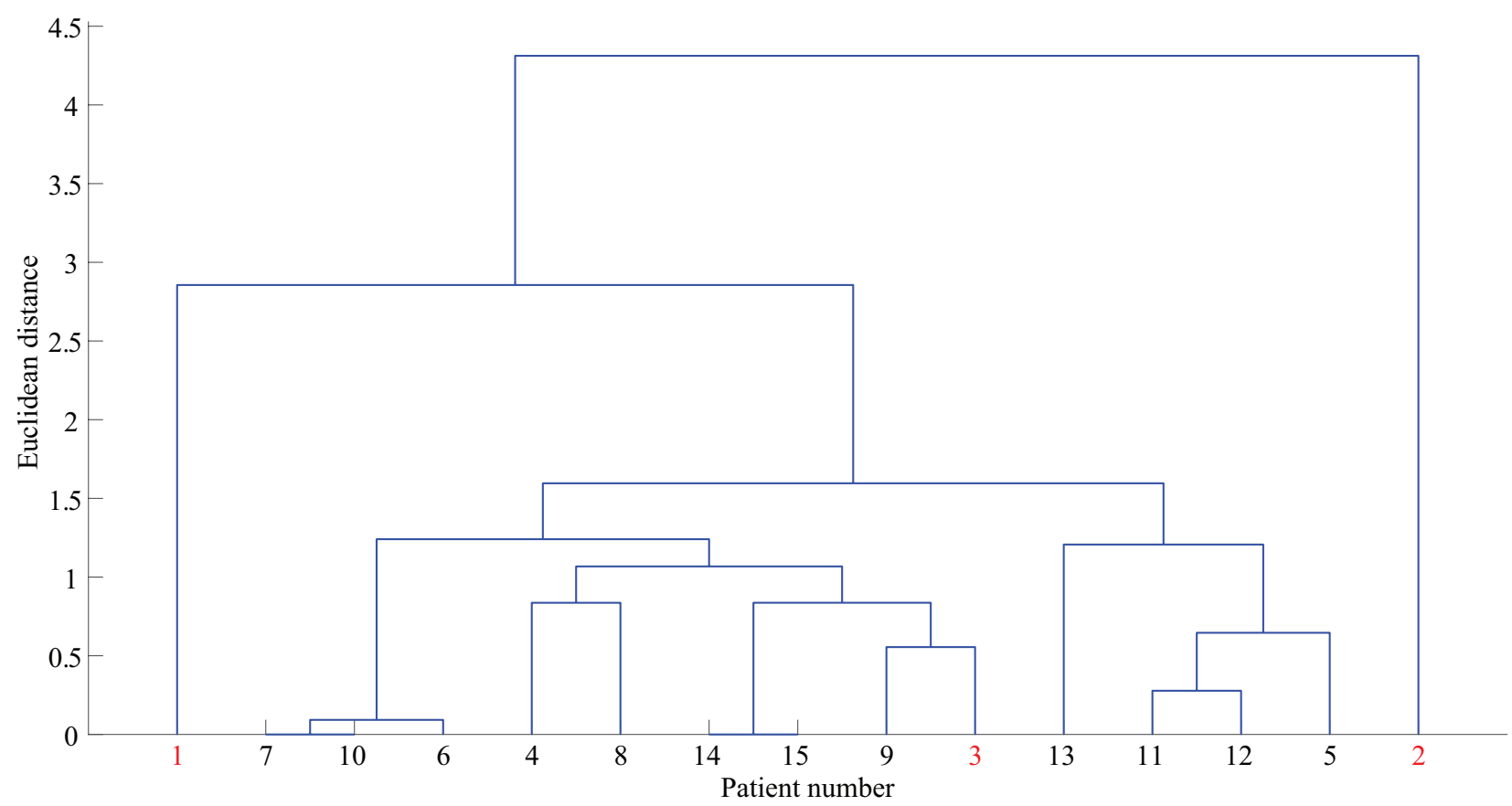

Figure 5. Dendrogram displaying patient categorization.

\subsection{Model Training and Prediction}

To amend the inequality between volunteers with ET and PT, as well as for increasing the available number of data and for providing an initial overview of their differential diagnosis and grouping, data augmentation techniques were considered. Data augmentation is often used in conjunction with convolutional neural networks (CNN) and can be used to address both the requirements of the diversity of the training data and the amount of data [57-60]. In addition to these two, augmented data can also be used to address the class imbalance problem in classification tasks, in our case the essential tremor class. Normally, in the case of many parameters, the learning model should be introduced with a proportional amount of examples to achieve satisfactory performance. To this goal, the data were initially separated into train and test data in order to be handled independently from each other and avoid information leakage from training to testing sets. Then, a sparse autoencoder with hidden size 1200 maximum epochs was trained in the Matlab environment along with a linear transfer function for the decoder. The autoencoder contains an autoencoder network, which consists of an encoder which maps the input to a hidden representation and a decoder which attempts to map this representation back to the original input. Specifically, the data were transferred to a low-level neural network that implements 
Principal Component Analysis (PCA) and, in this case, new data were predicted from the trained autoencoder [61]. It must be noted though that although the data augmentation technique aids to counter the limited data number and the class imbalance, it is by no means better than a database composed from real participants.

As we observe in Table 4, for each volunteer we received a total of 16 measurement subsets, as defined in Section 4.1. Therefore, tremor classification was performed in 16 different cases, concerning the 4 different arm poses and each of the 4 accelerometers, where the resulting acceleration vector was utilized for each case. Table 7 summarizes the number of original data collected, the data resulted from the data augmentation technique, as well as the total training and test data used for the classification procedure. For each of the three ET subjects (minority class) 15 new data were synthesized in order to counter the data imbalance. Each of the 8 PT subjects used for training had 5 new data synthesized while the rest 4 PT subjects used for testing had 3 new data synthesized. Finally, each of the 15 NT subjects who were categorized as training data had 3 new data synthesized and each of the rest 13 subjects sorted as test data had 1 new data synthesized.

Table 7. Summary of data used for classification.

\begin{tabular}{cccc}
\hline Data & Original (ET, PT, NT) & Augmented (ET, PT, NT) & Total (ET, PT, NT) \\
\hline Training & $25(2,8,15)$ & $115(30,40,45)$ & $140(32,48,60)$ \\
Test & $18(1,4,13)$ & $40(15,12,13)$ & $58(16,16,26)$ \\
Total & $43(3,12,28)$ & $155(45,52,58)$ & $198(48,64,86)$ \\
\hline
\end{tabular}

For the training examples, the 12 parameters extracted from the data processing technique were defined as predictors and $k$ as a response variable for tremor, with $k=1$ for ET, $k=2$ for PT and $k=3$ for NT volunteers. The training examples were a total of 140, with 32 belonging to the ET class, 48 to PT and the remaining 60 as NT cases. The 21 training algorithms were tested via Matlab and the respective training success rates were extracted for each of the measurements. The models with the higher accuracy were stored and were used for predictions.

For the test data, the tremor prediction rates were extracted for the respective machine learning algorithms selected for each measurement subset. The test data included a total of 58 examples, with the first 16 involving ET, the next $16 \mathrm{PT}$, while the remaining 26 examples involved NT cases.

\subsection{Results}

Table 8 lists the name of each measurement subset, the three best prediction algorithms for each of the 16 measurements, their success rates, both aggregated and separated for each type of tremor, as well as the training times and the prediction speeds of these classifiers. Variable success rates when trying to make predictions with new examples (test data) are observed.

Table 8. Maximum achieved prediction rates.

\begin{tabular}{|c|c|c|c|c|c|c|c|}
\hline Measurement & $\begin{array}{l}\mathrm{ET}(\%) \\
(k=1)\end{array}$ & $\begin{array}{l}\text { PT (\%) } \\
(k=2)\end{array}$ & NT (\%) $(k=3)$ & Total $(\%)$ & Algorithm & $\begin{array}{l}\text { Training Time } \\
\text { (sec) }\end{array}$ & $\begin{array}{l}\text { Prediction } \\
\text { Speed (obj./sec) }\end{array}$ \\
\hline \multirow{3}{*}{$I_{1}$} & 93.7 & 68.7 & 50.0 & 67.2 & RUS Boosted trees & 0.45 & 4800 \\
\hline & 87.5 & 37.5 & 73.1 & 67.2 & Medium tree & 4.71 & 3400 \\
\hline & 87.5 & 43.7 & 43.6 & 51.72 & Bagged trees & 7.29 & 690 \\
\hline \multirow{3}{*}{$I_{2}$} & 100.0 & 75.0 & 92.3 & 89.6 & Bagged trees & 2.89 & 1200 \\
\hline & 100.0 & 50 & 92.3 & 78.8 & Quadratic SVM & 2.65 & 2800 \\
\hline & 100.0 & 41.7 & 92.1 & 78.8 & Fine KNN & 3.07 & 3300 \\
\hline \multirow{3}{*}{$I_{3}$} & 81.2 & 56.2 & 61.5 & 63.8 & Quadratic SVM & 1.81 & 3600 \\
\hline & 62.5 & 45.8 & 73.1 & 61.4 & Linear SVM & 1.91 & 3800 \\
\hline & 62.5 & 37.5 & 73.1 & 57.6 & Bagged trees & 2.12 & 1300 \\
\hline
\end{tabular}


Table 8. Cont.

\begin{tabular}{|c|c|c|c|c|c|c|c|}
\hline Measurement & $\begin{array}{l}\mathrm{ET}(\%) \\
(k=1)\end{array}$ & $\begin{array}{l}\mathrm{PT}(\%) \\
(k=2)\end{array}$ & NT $(\%)(k=3)$ & Total (\%) & Algorithm & $\begin{array}{l}\text { Training Time } \\
\text { (sec) }\end{array}$ & $\begin{array}{l}\text { Prediction } \\
\text { Speed (obj./sec) }\end{array}$ \\
\hline \multirow{3}{*}{$I_{4}$} & 100.0 & 6.2 & 92.3 & 70.7 & Bagged trees & 2.18 & 1300 \\
\hline & 93.7 & 6.25 & 91.7 & 67.8 & Linear SVM & 1.66 & 1900 \\
\hline & 100.0 & 0 & 91.7 & 67.8 & Quadratic SVM & 1.58 & 3800 \\
\hline \multirow{3}{*}{$T_{1}$} & 100.0 & 50.0 & 96.1 & 84.5 & Fine Gaussian SVM & 1.72 & 2700 \\
\hline & 100.0 & 50.0 & 100.0 & 85.7 & Weighted KNN & 1.67 & 6300 \\
\hline & 93.7 & 36.4 & 95.8 & 78.8 & Quadratic SVM & 1.86 & 2700 \\
\hline \multirow{3}{*}{$T_{2}$} & 100.0 & 87.5 & 88.4 & 91.4 & Quadratic SVM & 1.88 & 4100 \\
\hline & 100.0 & 100.0 & 61.5 & 78.3 & Fine Gaussian SVM & 1.74 & 3500 \\
\hline & 100.0 & 100.0 & 30.78 & 68.9 & Linear SVM & 1.95 & 4300 \\
\hline \multirow{3}{*}{$T_{3}$} & 100.0 & 18.7 & 84.6 & 70.7 & Bagged trees & 2.28 & 1200 \\
\hline & 100.0 & 0 & 100.0 & 71.9 & Fine Gaussian SVM & 1.68 & 3500 \\
\hline & 100.0 & 0 & 28 & 40.3 & Linear SVM & 1.96 & 3500 \\
\hline \multirow{3}{*}{$T_{4}$} & 100.0 & 75.0 & 92.3 & 89.6 & Bagged trees & 2.83 & 950 \\
\hline & 100.0 & 0 & 100.0 & 73.9 & Quadratic SVM & 1.73 & 3300 \\
\hline & 100.0 & 0 & 100.0 & 73.9 & Linear SVM & 1.80 & 3600 \\
\hline \multirow{3}{*}{$M_{1}$} & 93.7 & 87.5 & 38.4 & 67.2 & Bagged trees & 2.26 & 1000 \\
\hline & 93.7 & 50 & 50 & 62.1 & Weighted KNN & 0.55 & 5700 \\
\hline & 93.7 & 56.25 & 50 & 63.2 & Quadratic SVM & 1.75 & 3000 \\
\hline \multirow{3}{*}{$M_{2}$} & 100.0 & 100.0 & 92.3 & 96.5 & Linear SVM & 1.86 & 3600 \\
\hline & 100.0 & 100.0 & 100.0 & 100.0 & Quadratic SVM & 1.78 & 3500 \\
\hline & 100.0 & 100.0 & 100.0 & 100.0 & Cubic SVM & 1.69 & 3600 \\
\hline \multirow{3}{*}{$M_{3}$} & 100.0 & 50.0 & 100.0 & 86.2 & Cubic SVM & 1.20 & 2900 \\
\hline & 0 & 75.0 & 100.0 & 62.5 & Quadratic SVM & 1.28 & 2900 \\
\hline & 0 & 75.0 & 100.0 & 62.5 & Linear SVM & 1.36 & 3700 \\
\hline \multirow{3}{*}{$M_{4}$} & 100.0 & 68.7 & 100.0 & 91.4 & Cubic SVM & 2.20 & 4700 \\
\hline & 100.0 & 68.7 & 100.0 & 91.4 & Quadratic SVM & 2.53 & 4300 \\
\hline & 100.0 & 62.5 & 92.3 & 86.2 & Fine KNN & 1.41 & 3800 \\
\hline \multirow{3}{*}{$F_{1}$} & 93.7 & 43.7 & 50.0 & 60.3 & Linear SVM & 1.89 & 4200 \\
\hline & 100.0 & 31.2 & 52.4 & 60.2 & Quadratic SVM & 1.81 & 3500 \\
\hline & 100.0 & 50.0 & 57.1 & 67.9 & Bagged trees & 2.46 & 890 \\
\hline \multirow{3}{*}{$F_{2}$} & 100.0 & 93.7 & 34.6 & 68.9 & Bagged trees & 2.69 & 1100 \\
\hline & 100.0 & 68.7 & 23.1 & 56.9 & Quadratic SVM & 2.45 & 4500 \\
\hline & 100.0 & 68.7 & 23.1 & 56.9 & Fine KNN & 1.98 & 5300 \\
\hline \multirow{3}{*}{$F_{3}$} & 100.0 & 75.0 & 76.9 & 82.8 & Quadratic discriminant & 1.65 & 6100 \\
\hline & 100.0 & 75.0 & 95.2 & 90.6 & Linear discriminant & 1.78 & 8600 \\
\hline & 100.0 & 50.0 & 100.0 & 84.9 & Linear SVM & 2.28 & 4100 \\
\hline \multirow{3}{*}{$F_{4}$} & 100.0 & 100.0 & 92.3 & 96.5 & Cubic SVM & 1.38 & 2200 \\
\hline & 100.0 & 100.0 & 86.9 & 92.0 & Linear SVM & 1.17 & 4200 \\
\hline & 100.0 & 75.0 & 93.3 & 90 & Bagged trees & 2.82 & 860 \\
\hline
\end{tabular}

From the presented results, there are algorithms that predicted correctly all 16 ET cases. Specifically, for the thumb and forearm measurements the majority of the chosen classifiers were able to accurately discriminate subjects with ET among the rest of the subjects, while for the index and metacarpal positions the prediction percentages of ET are high and reach up to $100 \%$.

On the contrary, it appears challenging to distinguish between PT and NT volunteers. This is reasonable since there were Parkinson's-diagnosed volunteers who showed no tremor during the measurements, which was verified in the subsequent processing of the acquired data. This was expected, as tremor may be one of the basic characteristics of the disease, but it is not a necessary and unique trait of the disease and it may not have a lasting nature.

Therefore, in a resting position, where the hand is not affected from the gravitational pull, it is possible to produce false results in the distinction PT from NT cases. This phenomenon was not observed in ET subjects, most of which were properly classified, due to the fact that all such volunteers experienced tremor at a resting position. Thus, after the appropriate processing of the signals and the extraction of the relevant parameters for the 3 ET subjects and for the signals created on the basis of the data augmentation technique, they were correctly classified as ET observations, which applied to all measurement positions. However, PT was correctly classified at 100\% for the SVM algorithms in both extended arm position measurements $\left(M_{2}, T_{2}\right)$ and in motion while holding an object $\left(F_{4}\right)$. 
From the algorithms with the highest success rate, the lowest achieved rate was $40.3 \%$ for the Linear SVM classifier. This percentage belonged to the thumb measurement in free motion, while also relatively low percentages were observed in measurements at resting positions of the index $\left(I_{1}\right.$-Bagged Trees), metacarpal $\left(M_{1}\right)$ and lower forearm ( $F_{1}$-Weighted $\left.\mathrm{KNN}\right)$ and at free motion measurements of the index ( $I_{3}$ - Bagged Trees) and thumb ( $T_{3}$-Linear SVM).

The highest success rates measured up to $100.0 \%$ for the Cubic and Quadratic SVM algorithms in extended arm position measurement of the metacarpal area $\left(M_{2}\right)$. Furthermore, the $96.5 \%$ accuracy provided by the Cubic SVM classifier in $F_{4}$ stands out among the rest in terms of accuracy while success rates over $90 \%$ were provided for other measurements in the aforementioned arm positions $\left(M_{4}\right.$-Cubic SVM, $T_{2}$-Quadratic SVM, $\left.T_{4}\right)$. In the object-holding arm position, the classification of new examples was more successful than of the free motion position, because as the hand moves, the strength of the muscles gradually weakens causing an increase in tremor amplitude.

In the midst of the extracted success rates are the tremor identification algorithms based on free movement positions. Although at these positions the hand muscles are similarly weakened due to the existence of gravitational pull and movement, they do not fade in free-motion as much as in the holding-object position and the characteristics of each tremor become less distinct. However, accuracy of around $90 \%$ can be observed for Cubic SVM and Linear discriminant through the free motion measurements of the metacarpal and lower forearm.

In order to further analyze the classifiers' performance, the training times and prediction speeds have been extracted and are presented in Table 8. As observed, the lowest training time was achieved by RUSBoosted trees classifier with $0.45 \mathrm{~s}$ during $I_{1}$ measurement, along with the high prediction speed of $4800 \mathrm{obj}$./s. However, RUSBoosted trees managed to classify correctly $67.2 \%$ of the total examples, a relatively low rate comparing to the rest of the classifiers. Similarly, Weighted KNN achieved high prediction speed (5700 obj./s) with low training time $(0.55 \mathrm{~s})$ but in expense of low accuracy rate, that of $62.1 \%$. It is noteworthy that the highest training time was over $7 \mathrm{~s}$ from Bagged trees during $I_{1}$, the same measurement where the lowest training time was achieved. In $M_{2}$, Quadratic and Cubic SVM classifiers achieved the highest accuracy rates while combining relatively low training time and high prediction speed. Finally, Linear SVM must be highlighted as the classifier that during $F_{4}$ measurement was characterized by high accuracy, training time and prediction speed with $92.0 \%, 1.17 \mathrm{~s}$ and $4200 \mathrm{obj} . / \mathrm{s}$, respectively.

Tables 9-12 summarize the average sensitivity, specificity and accuracy percentages of each measurement subset, in order to further assess the presented classification results concerning the performance of the 21 selected classifiers.

Table 9 presents the average values of sensitivity, specificity and accuracy percentages for each of the 21 tested classifiers for the rest measurement subsets. As observed from the Table, the majority of the algorithms were able to accurately classify the positive cases of ET with high success. Especially, all but one SVM classifier reached very high sensitivity percentages and even near 99\% for the case of Cubic SVM. In parallel, SVM algorithms managed to classify most of the negative cases of ET as negative, although similar to the sensitivity, for ET cases most of the classifiers achieved high specificity percentages with Medium KNN and Boosted trees classifiers predicting correctly all the negative ET cases as negative. Unlike the ET cases, for the PT we can discern the difficulty of the classifiers to correctly predict the positive cases with the maximum sensitivity percentage being that of Medium Gaussian SVM classifier with $65.63 \%$. On the contrary, the specificity rates are highly increased and reach up to $100 \%$ for Boosted trees. Lastly, regarding the sensitivity rates of NT, Boosted trees and Medium KNN classifiers achieved the highest with 100\% while for the specificity of NT, RUS Boosted trees achieved a percentage of $89.84 \%$. 
Table 9. Average sensitivity, specificity and accuracy percentages of classification methods for rest position measurements.

\begin{tabular}{lccccccc}
\hline \multirow{2}{*}{ Classifier } & \multicolumn{3}{c}{ Sensitivity } & \multicolumn{3}{c}{ Specificity } & \\
\cline { 2 - 7 } & ET & PT & NT & ET & PT & NT & \\
\hline Fine Tree & 90.63 & 51.56 & 46.52 & 85.59 & 73.92 & 81.25 & 60.31 \\
Medium Tree & 90.63 & 51.56 & 46.52 & 85.59 & 73.92 & 81.25 & 60.31 \\
Coarse Tree & 89.06 & 57.81 & 35.53 & 90.62 & 65.43 & 79.69 & 57.30 \\
Linear discriminant & 89.06 & 59.38 & 64.74 & 83.64 & 83.53 & 88.28 & 69.88 \\
Quadratic discriminant & 85.94 & 48.44 & 77.24 & 92.57 & 87.10 & 75.00 & 71.60 \\
Linear SVM & 96.88 & 39.06 & 76.01 & 80.98 & 94.64 & 80.47 & 71.54 \\
Quadratic SVM & 96.88 & 39.06 & 75.60 & 85.86 & 91.51 & 78.13 & 71.56 \\
Cubic SVM & 98.44 & 43.75 & 80.59 & 87.81 & 95.24 & 77.34 & 75.17 \\
Fine Gaussian SVM & 92.19 & 64.06 & 59.66 & 97.30 & 75.43 & 81.25 & 69.73 \\
Medium Gaussian SVM & 92.19 & 65.63 & 50.41 & 88.67 & 77.73 & 83.59 & 66.78 \\
Coarse Gaussian SVM & 48.44 & 32.81 & 64.29 & 82.74 & 75.97 & 64.84 & 50.98 \\
Fine KNN & 98.44 & 53.13 & 57.55 & 84.75 & 82.66 & 84.38 & 67.64 \\
Medium KNN & 84.38 & 62.50 & 47.02 & 88.84 & 72.38 & 82.03 & 62.08 \\
Coarse KNN & 0.00 & 0.00 & 100.00 & 100.00 & 98.21 & 2.34 & 43.53 \\
Cosine KNN & 93.75 & 53.13 & 40.75 & 86.42 & 67.29 & 86.72 & 59.26 \\
Cubic KNN & 79.69 & 62.50 & 47.25 & 90.19 & 69.00 & 82.03 & 60.66 \\
Weighted KNN & 96.88 & 62.50 & 50.14 & 90.70 & 76.54 & 82.81 & 66.86 \\
Boosted trees & 0.00 & 0.00 & 100.00 & 100.00 & 100.00 & 0.00 & 43.53 \\
Bagged Trees & 92.19 & 48.44 & 57.10 & 90.03 & 81.55 & 73.44 & 64.79 \\
Subspace KNN & 92.19 & 29.69 & 41.71 & 75.58 & 78.17 & 74.22 & 52.43 \\
RUS Boosted trees & 96.88 & 59.38 & 46.02 & 77.69 & 80.20 & 89.84 & 64.00 \\
\hline
\end{tabular}

Regarding the average accuracy for each of the 21 classifiers for the rest measurement subsets, the Cubic SVM classifier provided the most successful prediction rate with $75.17 \%$, while Linear, Quadratic and Fine Gaussian SVM reached relatively high percentages near $70 \%$. Moreover, Cubic SVM, as observed from the Table 9, appears to have high sensitivity and specificity rates, except the occasion of the PT sensitivity which is lower than $45 \%$.

Table 10 shows the average sensitivity, specificity and accuracy percentages for the measurement subset at postural position. Regarding the classification of ET, we can observe that 18 out of 21 classifiers were able to accurately predict the positive ET cases with a success rate over $96.88 \%$. In parallel, all the classifiers predicted correctly the negative cases as negative with percentages over $78.55 \%$. For the subjects with PT, the sensitivities of the classifiers at rest positions are clearly lower than the ones for the ET subjects. However, Quadratic discriminant classifier predicted correctly the $81.25 \%$ of positive cases of PT, although the specificity of the classifier for PT instances fell below $69 \%$. On the contrary, Boosted trees classifier managed to identify correctly all the negative cases of PT. Lastly, all the classifiers showed difficulty in predicting all NT positive cases, except the Boosted trees which achieved $100 \%$ sensitivity of NT cases despite the inability of the same classifier to predict any of the negative NT cases. Specifically, the majority of the tested classifiers categorized the negative NT cases as negative at a percentage around $90 \%$.

Regarding the average accuracy for each of the 21 classifiers for the postural measurement subset, Fine KNN classifier stands out as the most successful in terms of prediction rate with $72.81 \%$. Furthermore, this classifier, as observed from Table 10, appears to have high sensitivity and specificity rates, except the occasion of the NT sensitivity which is lower than $60 \%$. It must be mentioned that the accuracy rates of the most of the classifiers are close to $70 \%$ and the accuracy of Fine KNN. 
Table 10. Average sensitivity, specificity and accuracy percentages of classification methods for postural position measurements.

\begin{tabular}{lccccccc}
\hline \multirow{2}{*}{ Classifier } & \multicolumn{3}{c}{ Sensitivity } & \multicolumn{3}{c}{ Specificity } & \\
\cline { 2 - 7 } & ET & PT & NT & ET & PT & NT & \\
\hline Fine Tree & 98.44 & 54.69 & 58.23 & 83.53 & 81.36 & 90.63 & 68.15 \\
Medium Tree & 98.44 & 54.69 & 58.23 & 83.53 & 81.36 & 90.63 & 68.15 \\
Coarse Tree & 98.44 & 48.44 & 57.48 & 78.55 & 83.47 & 90.63 & 65.83 \\
Linear Discriminant & 98.44 & 53.13 & 64.24 & 84.41 & 81.20 & 96.09 & 71.59 \\
Quadratic Discriminant & 100.00 & 81.25 & 55.11 & 100.00 & 68.43 & 90.63 & 70.41 \\
Linear SVM & 96.88 & 53.13 & 48.15 & 88.51 & 66.30 & 93.75 & 61.68 \\
Quadratic SVM & 100.00 & 62.50 & 54.27 & 94.14 & 70.07 & 90.63 & 67.48 \\
Cubic SVM & 100.00 & 71.88 & 50.62 & 96.27 & 66.61 & 91.41 & 66.83 \\
Fine Gaussian SVM & 100.00 & 70.31 & 58.03 & 100.00 & 70.73 & 85.16 & 69.83 \\
Medium Gaussian SVM & 100.00 & 54.69 & 56.97 & 90.51 & 73.85 & 91.41 & 68.08 \\
Coarse Gaussian SVM & 67.19 & 50.00 & 61.16 & 91.51 & 69.27 & 85.16 & 62.95 \\
Fine KNN & 100.00 & 76.56 & 59.18 & 98.90 & 72.22 & 89.84 & 72.81 \\
Medium KNN & 100.00 & 51.56 & 60.21 & 88.51 & 76.82 & 92.19 & 69.32 \\
Coarse KNN & 0.00 & 18.75 & 82.69 & 100.00 & 79.76 & 21.88 & 50.30 \\
Cosine KNN & 100.00 & 43.75 & 55.67 & 81.85 & 77.08 & 93.75 & 65.29 \\
Cubic KNN & 100.00 & 46.88 & 59.84 & 87.01 & 77.13 & 91.41 & 68.07 \\
Weighted KNN & 100.00 & 51.56 & 61.36 & 90.51 & 76.65 & 90.63 & 69.98 \\
Boosted trees & 0.00 & 0.00 & 100.00 & 100.00 & 100.00 & 0.00 & 52.89 \\
Bagged Trees & 100.00 & 65.63 & 60.36 & 93.57 & 75.47 & 91.41 & 71.86 \\
Subspace KNN & 100.00 & 60.94 & 58.32 & 91.20 & 75.17 & 89.84 & 69.08 \\
RUS Boosted trees & 100.00 & 54.69 & 58.18 & 84.31 & 82.41 & 86.72 & 68.27 \\
\hline
\end{tabular}

Table 11. Average sensitivity, specificity and accuracy percentages of classification methods for free motion measurements.

\begin{tabular}{lccccccc}
\hline \multirow{2}{*}{ Classifier } & \multicolumn{3}{c}{ Sensitivity } & \multicolumn{3}{c}{ Specificity } & \\
\cline { 2 - 7 } & ET & PT & NT & ET & PT & NT & \\
\hline Fine Tree & 82.81 & 9.38 & 91.59 & 82.71 & 95.06 & 66.41 & 66.62 \\
Medium Tree & 82.81 & 9.38 & 91.59 & 82.71 & 95.06 & 66.41 & 66.62 \\
Coarse Tree & 78.13 & 3.13 & 92.26 & 79.05 & 97.56 & 63.28 & 64.36 \\
Linear Discriminant & 50.00 & 37.50 & 65.02 & 65.36 & 86.98 & 77.34 & 53.16 \\
Quadratic Discriminant & 50.00 & 4.69 & 99.26 & 100.00 & 89.48 & 40.63 & 60.28 \\
Linear SVM & 50.00 & 32.81 & 62.88 & 67.49 & 84.98 & 73.44 & 51.10 \\
Quadratic SVM & 57.81 & 39.06 & 91.59 & 93.01 & 88.48 & 65.63 & 68.13 \\
Cubic SVM & 57.81 & 15.63 & 84.84 & 92.34 & 80.30 & 60.16 & 57.95 \\
Fine Gaussian SVM & 50.00 & 0.00 & 100.00 & 100.00 & 100.00 & 25.00 & 59.52 \\
Medium Gaussian SVM & 65.63 & 28.13 & 73.58 & 94.95 & 85.86 & 49.22 & 58.12 \\
Coarse Gaussian SVM & 25.00 & 31.25 & 84.84 & 91.50 & 85.80 & 45.31 & 53.47 \\
Fine KNN & 65.63 & 7.81 & 84.12 & 93.82 & 95.71 & 36.72 & 57.44 \\
Medium KNN & 65.63 & 28.13 & 75.53 & 90.89 & 92.24 & 48.44 & 59.26 \\
Coarse KNN & 0.00 & 0.00 & 98.81 & 100.00 & 99.32 & 0.00 & 44.48 \\
Cosine KNN & 65.63 & 32.81 & 64.72 & 82.46 & 90.20 & 56.25 & 55.90 \\
Cubic KNN & 65.63 & 28.13 & 70.90 & 88.89 & 91.14 & 48.44 & 56.93 \\
Weighted KNN & 65.63 & 28.13 & 76.53 & 91.50 & 92.74 & 47.66 & 59.69 \\
Boosted trees & 0.00 & 0.00 & 100.00 & 100.00 & 100.00 & 0.00 & 44.32 \\
Bagged Trees & 90.63 & 12.50 & 80.29 & 78.85 & 93.56 & 69.53 & 63.53 \\
Subspace KNN & 71.88 & 29.69 & 70.55 & 96.82 & 82.06 & 54.69 & 59.48 \\
RUS Boosted trees & 57.81 & 9.38 & 93.06 & 86.25 & 90.56 & 57.81 & 60.48 \\
\hline
\end{tabular}


As shown in Table 11, ET sensitivity percentages for the measurement subset at free motion position are clearly lower than the rest and postural measurement subsets. More specifically, only the Trees classifiers achieved high sensitivity rates with only the Bagged trees reaching up to $90 \%$. However, this is not the case for ET specificity, where the majority of algorithms classified as negative the ET negative cases with success rates up to $100 \%$ for Quadratic discriminant analysis, Fine Gaussian SVM, Coarse KNN and Boosted trees. Furthermore, regarding the positive PT cases, only $39.06 \%$ of them were correctly categorized by the Quadratic SVM classifier while the other classifiers had even lower sensitivity percentages. In a different pattern, the PT specificity rates for the majority of the classifiers are over $90 \%$. with Fine Gaussian SVM and Boosted trees predicting correctly all the negative PT cases. Moreover, and in comparison to the positive PT cases, the NT ones were categorized as positive at high percentages with Fine Gaussian SVM and Boosted trees achieving the highest success rates. Lastly, the specificity rates of NT remained at relatively high levels with the best percentage being that of $77.34 \%$ from Linear Discriminant classifier.

In general, the accuracy percentages of the classifiers from the free motion measurement subset are lower than the ones extracted from the previous measurement subsets. This is an expected outcome, because, as seen from the aforementioned paragraph, the sensitivity and specificity rates were lower than for the rest and postural measurement subsets. Specifically, Quadratic SVM along with the Tree classifiers achieved the highest accuracy rates reaching up to $68.13 \%$.

Table 12. Average sensitivity, specificity and accuracy percentages of classification methods for motion with object measurements.

\begin{tabular}{lccccccc}
\hline \multirow{2}{*}{ Classifier } & \multicolumn{3}{c}{ Sensitivity } & \multicolumn{3}{c}{ Specificity } & \\
\cline { 2 - 7 } & ET & PT & NT & ET & PT & NT & \\
\hline Fine Tree & 82.81 & 9.38 & 91.59 & 82.71 & 95.06 & 66.41 & 66.62 \\
Medium Tree & 82.81 & 9.38 & 91.59 & 82.71 & 95.06 & 66.41 & 66.62 \\
Coarse Tree & 78.13 & 3.13 & 92.26 & 79.05 & 97.56 & 63.28 & 64.36 \\
Linear Discriminant & 100.00 & 35.94 & 73.53 & 82.96 & 86.61 & 86.72 & 70.71 \\
Quadratic Discriminant & 100.00 & 35.94 & 73.53 & 82.96 & 86.61 & 86.72 & 70.71 \\
Linear SVM & 100.00 & 35.94 & 70.65 & 82.96 & 84.82 & 86.72 & 69.42 \\
Quadratic SVM & 98.44 & 48.44 & 86.68 & 87.50 & 92.73 & 86.72 & 79.13 \\
Cubic SVM & 98.44 & 48.44 & 86.68 & 87.50 & 92.73 & 86.72 & 79.13 \\
Fine Gaussian SVM & 98.44 & 48.44 & 88.86 & 82.58 & 94.05 & 98.44 & 80.86 \\
Medium Gaussian SVM & 100.00 & 40.63 & 90.53 & 84.45 & 95.24 & 90.63 & 80.40 \\
Coarse Gaussian SVM & 100.00 & 40.63 & 91.91 & 83.26 & 97.02 & 93.75 & 82.62 \\
Fine KNN & 100.00 & 25.00 & 84.56 & 90.48 & 87.50 & 75.00 & 71.36 \\
Medium KNN & 100.00 & 37.50 & 85.72 & 79.98 & 94.94 & 93.75 & 78.47 \\
Coarse KNN & 85.94 & 50.00 & 85.98 & 83.18 & 89.29 & 99.22 & 78.00 \\
Cosine KNN & 100.00 & 37.50 & 87.75 & 85.42 & 94.35 & 86.72 & 79.01 \\
Cubic KNN & 98.44 & 39.06 & 77.32 & 76.33 & 90.48 & 100.00 & 74.37 \\
Weighted KNN & 0.00 & 3.13 & 100.00 & 100.00 & 97.32 & 6.25 & 56.40 \\
Boosted trees & 96.88 & 17.19 & 66.75 & 64.43 & 90.18 & 99.22 & 64.79 \\
Bagged Trees & 98.44 & 37.50 & 76.36 & 76.33 & 89.88 & 99.22 & 73.50 \\
Subspace KNN & 100.00 & 34.38 & 81.17 & 75.74 & 93.45 & 98.44 & 75.23 \\
RUS Boosted trees & 0.00 & 0.00 & 100.00 & 100.00 & 100.00 & 0.00 & 55.72 \\
\hline
\end{tabular}

The sensitivity, specificity and accuracy rates of the measurement subset related to motion while holding an object are summarized at Table 12. By looking at the table, the majority of the classifiers appear to have high ET sensitivity rates up to $100 \%$. The same can be said for the ET specificity and for the Weighted KNN and RUSBoosted trees classifiers. However, it is worth mentioning that these two classifiers with the highest ET specificity have the lowest ET sensitivity as they were unable to categorize a single positive ET case 
as positive. Moreover, the correct classification of the positive PT cases proved difficult for the classifiers with the highest percentages being below 50\% which was the best PT sensitivity rate achieved from the Coarse KNN. On the other hand, the algorithms were able to categorize as negative most of negative PT cases with the RUSBoosted trees classifier achieving $100 \%$ success rate. Lastly, both the NT sensitivity and specificity rates are high for most of the classifiers with Weighted KNN and RUSBoosted trees having 100\% sensitivity percentage and Cubic KNN having the same perfect specificity percentage.

Regarding the average accuracy for each of the 21 classifiers for the motion with object measurement, subset Coarse Gaussian SVM classifier stands out as the most successful in terms of accuracy with $82.62 \%$ while simultaneously having high sensitivity and specificity rates, except NT sensitivity which is relatively low. Finally, it is notable that 5 out of 6 SVM classifiers predicted correctly the examples with approximately $80 \%$ success.

Overall, as we observe from Tables 9-12, ET is well classified for all four measurement subsets with high sensitivity and specificity percentages. On the contrary, positive PT cases were more difficult to classify. In detail, only in the postural measurement subset the positive cases of PT were correctly categorized as positive at a satisfactory level with the high success sensitivity rate of $81.25 \%$ from the Quadratic Discriminant classifier. However, the majority of the classifiers managed to efficiently categorize correctly the negative cases of PT at all four measurement positions and at percentages reaching up to $100 \%$ in each of the sets. In general, the NT cases were classified correctly at high success rates especially for free motion and motion with object measurement subsets. Regarding the various classifiers, Boosted trees recognized all the positive NT cases as positive at rest, postural and free motion measurement positions. Furthermore, the NT specificity rate was relatively high in all sets, but the motion with object one proved to be the most accurate with $100 \%$ specificity rate for Cubic KNN.

To summarize, the measurement subset which provided the best accuracy percentage is the motion while holding an object with $82.62 \%$ average accuracy for the accelerometer signals from index, thumb, metacarpal and forearm. This percentage is approximately $8 \%$ better than the second best accuracy achieved at rest position, $10 \%$ better than at postural position and $15 \%$ better than at free motion position. The highest percentage belongs to the Coarse Gaussian SVM classifier while the second best accuracy percentage belongs to Cubic SVM classifier at the rest measurement subset, with Fine KNN at postural position and Quadratic SVM at free motion position following third and forth, respectively. Therefore, our investigation shows that it is possible to efficiently classify among essential, Parkinson's and no diagnosed tremor, especially at motion with object position. This observation agrees with our findings from Table 8 that during the object-holding arm position, the classification of new examples is more successful than of the other three positions, for the reason that as the hand moves, the strength of the muscles gradually weakens causing an increase in tremor amplitude thus creating a clear margin of separation between the three classes.

\section{Conclusions}

In this work, an attempt was made in differentiating Parkinson's and essential tremor via the use of machine learning modeling algorithms. To this goal, a clinical trial was conducted involving tremor-diagnosed patients and healthy volunteers, where a data acquisition setup was implemented and utilized for acquiring acceleration measurements from multiple arm points and for multiple motion scenarios. Initially, after collecting data from the medical history of tremor-diagnosed patients, grouping of patients was attempted via the use of dendrograms, for investigating the possibility of distinguishing between these kinds of tremor via diagrammatic representation. The overlapping in symptoms and characteristics of these two types of tremor further highlighted the challenge and risk of their misdiagnosis, which fueled the utilization of more advanced machine learning techniques for improving the identification possibilities.

Via the acceleration measurements acquired from all volunteers under different arm poses and measurement points on the hand and forearm, the data was processed and 
augmented to account for the inequality between volunteers with essential and Parkinson's tremor, which was then followed by the extraction of statistical signal characteristics. Machine learning models were trained via the use of the augmented data, while multiple classification algorithms were tested for identifying the ones with the highest success rates and for making predictions.

From the tremor identification results, it was noted that the prediction algorithms with the lowest prediction percentages appeared in the measurements with the arm in a resting position or during free motion with the lowest achieved success rate was the of $T_{3}$ with $40.3 \%$. On the contrary, the highest forecasting percentages were acquired from SVM-based algorithms, in both extended and moving while holding an object arm poses. Specifically, Cubic and Quadratic SVM managed to predict correctly with high speed, while requiring low training time, all the examples at $M_{2}$. In parallel, Cubic SVM achieved 96.5\% success rate at $F_{4}$ while during the same measurement Linear SVM effectively combined accuracy with training time and prediction speed. Thus, the increased tremor in these two positions made the discrimination more distinct from each type of tremor parameter, thus resulting in more accurate predictions. In contrast, the free-motion arm pose, which includes the parallel action of different muscle groups, resulted in less muscle fatigue and less distinct characteristics for better discriminating among tremor types. In overall, Cubic and Quadratic SVM algorithms managed to provide good prediction rates under most measurement points and arm poses, reaching up to $100 \%$ prediction success rates for both (i) metacarpal measurements with the arm extended and (ii) forearm measurements with the arm moving while holding an object.

Finally, from the results presenting the average sensitivity, specificity and accuracy of each measurement subset for the 21 selected classifiers, the SVM algorithms provided the best results, where for the motion with object position the accuracy percentage of Coarse Guassian SVM reached up to that of $82.62 \%$. This high percentage along with superiority of the SVM classifiers denotes that there can be clear margin of separation between the three classes PT, ET and NT.

\section{Future Work}

The preliminary results presented in this article show the potential of utilizing machine learning optimization for discriminating between essential and Parkinson's tremor, while reinforcing the imperative need for future research. The future goal of the presented work is the development of an automated wearable diagnostic tool for early stages of different tremor syndromes, thus reducing the risk of a misdiagnosis and enabling an efficient and individualized treatment plan. Efforts to acquire data from a larger number of volunteers is an ongoing task, for the purpose of further improving the quality of the results, while configuring the measurement protocol to better facilitate tremor identification. Finally, the inclusion of volunteers with more tremor types, for expanding this research direction towards the creation of a differential diagnostic tool, as well as the tool's integration with tremor suppression methods involving soft exoskeletons [62] are also part of current and future work.

Author Contributions: Conceptualization, G.A. and V.S.; methodology, V.S., G.A. and Z.K.; software, V.S.; validation, V.S.; formal analysis, V.S., G.A.; investigation, V.S.; resources, G.A., Z.K. and P.P.; data curation, V.S.; writing —original draft preparation, V.S.; writing-review and editing, G.A., Z.K. and P.P.; visualization, G.A.; supervision, G.A. All authors have read and agreed to the published version of the manuscript.

Funding: This research received no external funding.

Institutional Review Board Statement: The study was conducted according to the guidelines of the Declaration of Helsinki, and approved by the Institutional Review Board of University of Patras (protocol code 13976, 3/10/2019).

Informed Consent Statement: Informed consent was obtained from all subjects involved in the study. 
Data Availability Statement: The data presented in this study are openly available in Zenodo at 10.5281 /zenodo.4698248.

Conflicts of Interest: The authors declare no conflict of interest.

$\begin{array}{ll}\text { Abbreviations } \\ \text { The following abbreviations are used in this manuscript: } \\ \text { PT } & \text { Parkinson's tremor } \\ \text { ET } & \text { Essential tremor } \\ \text { NT } & \text { No diagnosed tremor } \\ \text { TETRAS } & \text { The ET Rating Assessment Scale } \\ \text { FDA } & \text { Food and Drug Administration } \\ \text { PET } & \text { Positron Emission Tomography } \\ \text { CT } & \text { Computed tomography } \\ \text { MDS } & \text { Movement Disorder Society } \\ \text { UPDRS } & \text { Unified Parkinson's Disease Rating Scale } \\ \text { SPI } & \text { Serial Peripheral Interface } \\ \text { SSC } & \text { Statistical Signal Characteristics } \\ \text { LDA } & \text { Linear discriminant analysis } \\ \text { QDA } & \text { Quadratic discriminant analysis } \\ \text { SVM } & \text { Support vector machine } \\ \text { KNN } & \text { K-nearest neighbor } \\ \text { PSD } & \text { Power spectral density } \\ \text { CNN } & \text { Convolutional Neural Network } \\ \text { PCA } & \text { Principal Component Analysis }\end{array}$

\section{References}

1. Anouti, A.; Koller, W.C. Tremor disorders. Diagnosis and management. West. J. Med. 1995, 162, 510. [PubMed]

2. Bötzel, K.; Tronnier, V.; Gasser, T. Differenzialdiagnose und therapie des tremors. Dtsch. Arztebl 2014, 111, 225-235. [CrossRef]

3. Louis, E.D. Linking Essential Tremor to the Cerebellum: Neuropathological Evidence. Cerebellum 2016, 15, 235-242. [CrossRef]

4. Louis, E.D.; Ottman, R. How many people in the USA have essential tremor? Deriving a population estimate based on epidemiological data. Tremor Other Hyperkinet. Mov. 2014, 4, 259. [CrossRef] [PubMed]

5. McAuley, J.H. Physiological and pathological tremors and rhythmic central motor control. Brain 2000, 123, 1545-1567. [CrossRef] [PubMed]

6. Ahlrichs, C.; Lawo, M. Parkinson's Disease Motor Symptoms in Machine Learning: A Review. Health Inform. Int. J. 2013, 2, 1-18. [CrossRef]

7. Mandal, I.; Sairam, N. New machine-learning algorithms for prediction of Parkinson's disease. Int. J. Syst. Sci. 2014, 45, 647-666. [CrossRef]

8. Kavindya, P.; Awantha, W.V.; Wanasinghe, A.T.; Kulasekera, A.L.; Chathuranga, D.S.; Senanayake, B. Evaluation of Hand Tremor Frequency among Patients in Sri Lanka using a Soft Glove. In Proceedings of the MERCon 2020—6th International Multidisciplinary Moratuwa Engineering Research Conference, Moratuwa, Sri Lanka, 28-30 July 2020; Institute of Electrical and Electronics Engineers Inc.: New York, NY, USA, 2020; pp. 301-306. [CrossRef]

9. Hernqvist, C.; Rosander, M. Machine Learning for Symptoms Quantification of Parkinson's Disease Patients. Ph.D. Thesis, Chalmers University of Technology, Gothenburg, Sweden, 2017.

10. McGurrin, P.; McNames, J.; Wu, T.; Hallett, M.; Haubenberger, D. Quantifying Tremor in Essential Tremor using Inertial Sensors-Validation of an Algorithm. IEEE J. Transl. Eng. Health Med. 2020, 15, 25055-25071. [CrossRef]

11. Olsson, F.; Medvedev, A. Nonparametric Time-Domain Tremor Quantification with Smart Phone for Therapy Individualization. IEEE Trans. Control Syst. Technol. 2020, 28, 118-129. [CrossRef]

12. Dai, H.; Cai, G.; Lin, Z.; Wang, Z.; Ye, Q. Validation of Inertial Sensing-based Wearable Device for Tremor and Bradykinesia Quantification. IEEE J. Biomed. Health Inform. 2020, 1. [CrossRef]

13. Wu, H.; Zhang, Y.; Wu, X.; Yang, F. Assessment of Upper Limb Tremors in Patients with Parkinson's Disease Based on Displacement and Acceleration Information. In Proceedings of the 2020 5th IEEE International Conference on Automation, Control and Robotics Engineering (CACRE), Dalian, China, 19-20 September 2020; pp. 177-182. [CrossRef]

14. Duque, J.D.; Egea, A.J.; Reeb, T.; Rojas, H.A.; Gonzalez-Vargas, A.M. Angular Velocity Analysis Boosted by Machine Learning for Helping in the Differential Diagnosis of Parkinson's Disease and Essential Tremor. IEEE Access 2020, 8, 88866-88875. [CrossRef] 
15. Papadopoulos, A.; Kyritsis, K.; Klingelhoefer, L.; Bostanjopoulou, S.; Chaudhuri, K.R.; Delopoulos, A. Detecting Parkinsonian Tremor from IMU Data Collected In-The-Wild using Deep Multiple-Instance Learning. IEEE J. Biomed. Health Inform. 2019, 24, 2559-2569. [CrossRef]

16. Almeida, J.; Pedrosa, P.; Filho, R.; Carneiro, T.; Wei, W.; Damaševičius, R.; Maskeliūnas, R.; De, V.H.C.; Almeida, J.; Pedrosa, P.; et al. Detecting Parkinson's Disease with Sustained Phonation and Speech Signals using Machine Learning Techniques. In Pattern Recognition Letters; Elsevier: Amsterdam, The Netherlands, 2019; Volume 125, pp. 55-62.

17. Varghese, B.K.; Geraldine Bessie Amali, D.; Uma Devi, K.S. Prediction of parkinson's disease using machine learning techniques on speech dataset. Res. J. Pharm. Technol. 2019, 12, 1-5. [CrossRef]

18. Klempír, O.; Krupička, R. Machine learning using speech utterances for parkinson disease detection. Lékař a Technika 2018, $48,66-71$.

19. Wroge, T.J.; Ozkanca, Y.; Demiroglu, C.; Si, D.; Atkins, D.C.; Ghomi, R.H. Parkinson' s Disease Diagnosis Using Machine Learning and Voice. In Proceedings of the 2018 IEEE Signal Processing in Medicine and Biology Symposium (SPMB), Philadelphia, PA, USA, 1 December 2018; pp. 1-7.

20. Sriram, T.V.; Rao, M.V.; Satya Narayana, G.; Kaladhar, D. Diagnosis of Parkinson Disease Using Machine Learning and Data Mining Systems from Voice Dataset. Adv. Intell. Syst. Comput. 2014, 327, 151-157. [CrossRef]

21. Ray, S. A Predictive Diagnosis for Parkinson' s Disease Through Machine Learning. Can. Sci. Fair J. 2019, 2, 5-16.

22. Schaap, T. Predicting Severity of Parkinson's Disease with Typing Behavior: A Machine Learning Approach. Ph.D. Thesis, Universiteit Utrecht, Utrecht, The Netherlands, 2018.

23. Youngmann, B.; Allerhand, L.; Paltiel, O.; Yom-Tov, E.; Arkadir, D. A machine learning algorithm successfully screens for Parkinson's in web users. Ann. Clin. Transl. Neurol. 2019, 6, 2503-2509. [CrossRef] [PubMed]

24. Shiiba, T.; Arimura, Y.; Nagano, M.; Takahashi, T.; Takaki, A. Improvement of classification performance of Parkinson's disease using shape features for machine learning on dopamine transporter single photon emission computed tomography. PLoS ONE 2020, 15, e0228289. [CrossRef] [PubMed]

25. Ahmadi Rastegar, D.; Ho, N.; Halliday, G.M.; Dzamko, N. Parkinson's progression prediction using machine learning and serum cytokines. NPJ Parkinson's Dis. 2019, 5, 1-8. [CrossRef]

26. Tsoulos, I.G.; Mitsi, G.; Stavrakoudis, A.; Papapetropoulos, S. Application of machine learning in a parkinson's disease digital biomarker dataset using Neural Network Construction (NNC) methodology discriminates patient motor status. Front. ICT 2019, 6, 1-7. [CrossRef]

27. Pham, T.D.; Wardell, K.; Eklund, A.; Salerud, G. Classification of short time series in early Parkinson's disease with deep learning of fuzzy recurrence plots. IEEE/CAA J. Autom. Sin. 2019, 6, 1306-1317. [CrossRef]

28. Goschenhofer, J.; Pfister, F.M.; Yuksel, K.A.; Bischl, B.; Fietzek, U.; Thomas, J. Wearable-based Parkinson's Disease Severity Monitoring using Deep Learning. In Machine Learning and Knowledge Discovery in Databases, Proceedings of the Joint European Conference on Machine Learning and Knowledge Discovery in Databases, Würzburg, Germany, 16-20 September 2019; Springer: Berlin, Germany, 2019.

29. Ibrahim, A.; Zhou, Y.; Jenkins, M.E.; Luisa Trejos, A.; Naish, M.D. The Design of a Parkinson's Tremor Predictor and Estimator Using a Hybrid Convolutional-Multilayer Perceptron Neural Network. In Proceedings of the Annual International Conference of the IEEE Engineering in Medicine and Biology Society, Montréal, QC, Canada, 20-24 July 2021; Institute of Electrical and Electronics Engineers Inc.: New York, NY, USA, 2020; pp. 5996-6000. [CrossRef]

30. Wingate, J.; Kollia, I.; Bidaut, L.; Kollias, S. A Unified Deep Learning Approach for Prediction of Parkinson's Disease. IET Res. J. 2019, 14, 1-10.

31. Pereira, C.R.; Weber, S.A.; Hook, C.; Rosa, G.H.; Papa, J.P. Deep learning-aided Parkinson's disease diagnosis from handwritten dynamics. In Proceedings of the 2016 29th SIBGRAPI Conference on Graphics, Patterns and Images, SIBGRAPI 2016, Sao Paulo, Brazil, 4-7 October 2016; pp. 340-346. [CrossRef]

32. Grover, S.; Bhartia, S.; Yadav, A.; Seeja, K.R. Predicting Severity of Parkinson's Disease Using Deep Learning. Procedia Comput. Sci. 2018, 132, 1788-1794. [CrossRef]

33. Hossen, A.; Muthuraman, M.; Al-Hakim, Z.; Raethjen, J.; Deuschl, G.; Heute, U. Discrimination of Parkinsonian tremor from essential tremor using statistical signal characterization of the spectrum of accelerometer signal. Bio-Med. Mater. Eng. 2013, 23, 513-531. [CrossRef]

34. Darnall, N.D.; Donovan, C.K.; Aktar, S.; Tseng, H.Y.; Barthelmess, P.; Cohen, P.R.; Lin, D.C. Application of machine learning and numerical analysis to classify tremor in patients affected with essential tremor or Parkinson's disease. Gerontechnology 2012, 10, 208-219. [CrossRef]

35. Bartlett, C.; Miles, J.; Qamar, N.; Martinez, L.; Manno, D. TremorTrack: A privacy-aware solution for monitoring parkinsonian and essential tremors. In Proceedings of the IEEE Symposium on Computer-Based Medical Systems, Rochester, MN, USA, 28-30 July 2020; Institute of Electrical and Electronics Engineers Inc.: New York, NY, USA, 2020; pp. 392-396. [CrossRef]

36. Skaramagkas, V.; Andrikopoulos, G.; Kefalopoulou, Z.; Polychronopoulos, P. Towards Differential Diagnosis of Essential and Parkinson's Tremor via Machine Learning. In Proceedings of the 2020 28th Mediterranean Conference on Control and Automation (MED), Saint-Raphaël, France, 15-18 September 2020; pp. 782-787. [CrossRef]

37. Essential Tremor Disorder I Johns Hopkins Medicine. Available online: https://www.hopkinsmedicine.org/health/conditionsand-diseases / essential-tremor-disorder (accessed on 24 March 2020). 
38. Sullivan, K.L.; Zesiewicz, T. Overview of Essential Tremor. Neuropsychiatr. Dis. Treat. 2010, 6, 401-408. [CrossRef]

39. Lee, H.J.; Lee, W.W.; Kim, S.K.; Park, H.; Jeon, H.S.; Kim, H.B.; Jeon, B.S.; Park, K.S. Tremor frequency characteristics in Parkinson's disease under resting-state and stress-state conditions. J. Neurol. Sci. 2016, 362, 272-277. [CrossRef]

40. Cohen, O.; Pullman, S.; Jurewicz, E.; Watner, D.; Louis, E.D. Rest tremor in patients with essential tremor: Prevalence, clinical correlates, and electrophysiologic characteristics. Arch. Neurol. 2003, 60, 405-410. [CrossRef] [PubMed]

41. Clark, L.N.; Louis, E.D. Essential tremor. In Handbook of Clinical Neurology; Elsevier B.V.: Amsterdam, The Netherlands, 2018; Volume 147, pp. 229-239. [CrossRef]

42. Elble, R.J. The Essential Tremor Rating Assessment Scale. J. Neurol. Neuromed. 2016, 1, 34-38.

43. Goetz, C.G. The history of Parkinson's disease: Early clinical descriptions and neurological therapies. Cold Spring Harb. Perspect. Med. 2011, 1. [CrossRef] [PubMed]

44. Hallett, M. Parkinsonism and Related Disorders Parkinson's disease tremor: Pathophysiology. Park. Realt. Disord. 2012, 18, S85-S86. [CrossRef]

45. Parkinson's Disease PET Scans: Uses, Results, and More; WebMD LLC: New York, NY, USA, 2020.

46. Ling, H. Clinical Approach to Progressive Supranuclear Palsy. J. Mov. Disord. 2016, 9, 3-13. [CrossRef] [PubMed]

47. Calisto, A.; Bramanti, A.; Galeano, M.; Angileri, F.; Campobello, G.; Serrano, S.; Azzerboni, B. A preliminary study for investigating idiopatic normal pressure hydrocephalus by means of statistical parameters classification of intracranial pressure recordings. In Proceedings of the 31st Annual International Conference of the IEEE Engineering in Medicine and Biology Society: Engineering the Future of Biomedicine, EMBC 2009, Minneapolis, MN, USA, 3-6 September 2009; pp. 2629-2632. [CrossRef]

48. Anand, S.S.; Singh, H.; Dash, A.K. Clinical applications of PET and PET-CT. Med. J. Armed. Forces India 2009, 65, 353-358. [CrossRef]

49. Clarke, C.E.; Patel, S.; Ives, N.; Rick, C.E.; Woolley, R.; Wheatley, K.; Walker, M.F.; Zhu, S.; Kandiyali, R.; Yao, G.; et al. Physiotherapy and Occupational Therapy vs No Therapy in Mild to Moderate Parkinson Disease: A Randomized Clinical Trial. JAMA Neurol. 2016, 73. [CrossRef]

50. Schwab and England-Parkinson's Disease Research, Education and Clinical Centers; Talkspace Network LLC: New York, NY, USA, 2015.

51. Murtagh, F. Counting Dendrograms: A Survey. Discret. Appl. Math. 1984, 7, 191-199. [CrossRef]

52. Navada, A.; Ansari, A.N.; Patil, S.; Sonkamble, B.A. Overview of use of decision tree algorithms in machine learning. In Proceedings of the 2011 IEEE Control and System Graduate Research Colloquium, ICSGRC 2011, Shah Alam, Malaysia, 27-28 June 2011; pp. 37-42. [CrossRef]

53. Lin, G.C.; Wang, C.M.; Wang, W.J. An unsupervised linear discriminant analysis approach to multispectral MRI images classification. In Proceedings of the Sixth International Conference on Machine Learning and Cybernetics, ICMLC 2007, Hong Kong, China, 19-22 August 2007; Volume 4, pp. 2018-2023. [CrossRef]

54. Schölkopf, B. SVMs-A practical consequence of learning theory. IEEE Intell. Syst. Their Appl. 1998, 13, 18-21. [CrossRef]

55. Moldagulova, A.; Sulaiman, R.B. Using KNN algorithm for classification of textual documents. In Proceedings of the ICIT 2017-8th International Conference on Information Technology, Amman, Jordan, 17-18 May 2017; Institute of Electrical and Electronics Engineers Inc.: New York, NY, USA, 2017; pp. 665-671. [CrossRef]

56. Huang, F.; Xie, G.; Xiao, R. Research on ensemble learning. In Proceedings of the 2009 International Conference on Artificial Intelligence and Computational Intelligence, AICI 2009, Shanghai, China, 7-8 November 2009; Volume 3, pp. 249-252. [CrossRef]

57. Mikołajczyk, A.; Grochowski, M. Data augmentation for improving deep learning in image classification problem. In Proceedings of the 2018 International Interdisciplinary PhD Workshop, IIPhDW 2018, Swinoujscie, Poland, 9-12 May 2018; Institute of Electrical and Electronics Engineers Inc.: New York, NY, USA, 2018; pp. 117-122. [CrossRef]

58. Parente, A.P.; de Souza, M.B.; Valdman, A.; Folly, R.O.M. Data augmentation applied to machine learning-based monitoring of a pulp and paper process. Processes 2019, 7, 958. [CrossRef]

59. Barua, S.; Islam, M.M.; Yao, X.; Murase, K. MWMOTE-Majority weighted minority oversampling technique for imbalanced data set learning. IEEE Trans. Knowl. Data Eng. 2014, 26, 405-425. [CrossRef]

60. Guo, C.; Ma, Y.; Xu, Z.; Cao, M.; Yao, Q. An Improved Oversampling Method for Imbalanced Data-SMOTE Based on Canopy and K-Means; Institute of Electrical and Electronics Engineers Inc.: New York, NY, USA, 2019; pp. 1467-1469. [CrossRef]

61. Wang, W.; Huang, Y.; Wang, Y.; Wang, L. Generalized autoencoder: A neural network framework for dimensionality reduction. In Proceedings of the IEEE Computer Society Conference on Computer Vision and Pattern Recognition Workshops, Columbus, OH, USA, 23-28 June 2014; pp. 496-503. [CrossRef]

62. Skaramagkas, V.; Andrikopoulos, G.; Manesis, S. An Experimental Investigation of Essential Hand Tremor Suppression via a Soft Exoskeletal Glove. In Proceedings of the 2020 European Control Conference (ECC), Saint Petersburg, Russia, 12-15 May 2020; pp. 889-894. [CrossRef] 